\title{
REVIEWS
}

(O) TECHNIQUES, TECHNOLOGY \& ANALYSIS

\section{Bioelectric neuromodulation for gastrointestinal disorders: effectiveness and mechanisms}

\author{
Sophie C. Payne $\mathbb{B}^{1,2 *}$, John B. Furness $\mathbb{D}^{3,4}$ and Martin J. Stebbing ${ }^{3,4}$
}

Abstract | The gastrointestinal tract has extensive, surgically accessible nerve connections with the central nervous system. This provides the opportunity to exploit rapidly advancing methods of nerve stimulation to treat gastrointestinal disorders. Bioelectric neuromodulation technology has considerably advanced in the past decade, but sacral nerve stimulation for faecal incontinence currently remains the only neuromodulation protocol in general use for a gastrointestinal disorder. Treatment of other conditions, such as IBD, obesity, nausea and gastroparesis, has had variable success. That nerves modulate inflammation in the intestine is well established, but the anti-inflammatory effects of vagal nerve stimulation have only recently been discovered, and positive effects of this approach were seen in only some patients with Crohn's disease in a single trial. Pulses of high-frequency current applied to the vagus nerve have been used to block signalling from the stomach to the brain to reduce appetite with variable outcomes. Bioelectric neuromodulation has also been investigated for postoperative ileus, gastroparesis symptoms and constipation in animal models and some clinical trials. The clinical success of this bioelectric neuromodulation therapy might be enhanced through better knowledge of the targeted nerve pathways and their physiological and pathophysiological roles, optimizing stimulation protocols and determining which patients benefit most from this therapy.

Central nervous system (CNS). The nervous system consisting of the brain and spinal cord.
${ }^{1}$ Bionics Institute, East Melbourne, Victoria, Australia.

${ }^{2}$ Medical Bionics Department University of Melbourne, Parkville, Victoria, Australia.

${ }^{3}$ Florey Institute of Neuroscience and Mental Health, Parkville, Victoria, Australia

${ }^{4}$ Department of Anatomy and Neuroscience, University of Melbourne, Parkville,

Victoria, Australia.

*e-mail:spayne@

bionicsinstitute.org

https://doi.org/10.1038/

s41575-018-0078-6
The use of electrical stimulation of nerves or brain centres as a therapeutic tool is being trialled in an increasing range of human diseases, including Parkinson disease, arthritis, depressive illness, schizophrenia, pain and bladder dysfunction. This treatment approach is referred to variously as, simply, neuromodulation, or as bioelectric neuromodulation or electroceuticals. The possibility that bioelectric neuromodulation could be used to relieve gastrointestinal-related disorders has created considerable interest in the clinical community, and stimulation at a range of sites of the gastrointestinal tract for several different conditions has been investigated (FIG. 1). In most cases, outcomes have been variable and mechanisms of action remain unclear, leaving important therapeutic questions unanswered (BOX 1), for example, optimal stimulus parameters, which have been reviewed elsewhere ${ }^{1}$. Possible target populations and strategies for neuromodulation therapies applied to the gut have also been reviewed ${ }^{1-5}$. We believe that a clear understanding of the biology of gastrointestinal innervation is required to maximize the effectiveness and to minimize adverse effects of bioelectric neuromodulation therapies.

In this Review, we relate bioelectric neuromodulation therapies to knowledge of the nerve circuitry and discuss possible mechanisms that are engaged by the stimulation. We first review the known functions of the extrinsic nerves that are the main targets for bioelectric modulation of gastrointestinal function followed by the disorders that have been targeted, notably IBD, postoperative ileus, obesity, gastroparesis, nausea and colorectal dysfunction. For each disorder, we discuss the rationale for the use of bioelectric neuromodulation, reasons for failures or limited success and ways in which treatments might be improved.

\section{Extrinsic gastrointestinal innervation}

The digestive system receives innervation from the central nervous system (CNS) through the vagus nerves, thoracolumbar pathways and the sacral nerves. Neurons in these pathways innervate the enteric nervous system (ENS) and final effector systems, such as the sphincter muscle and intramural blood vessels, to influence various functions, including muscle movement, acid secretion, enteric hormone release, immune cell activity, inflammation and blood flow (FIG. 2). Sensory information from the digestive tract is communicated to the CNS through each of these pathways. The sensory neurons carry information about a wide range of conditions of the gut, 


\author{
Key points \\ - The gastrointestinal tract has substantial two-way neural interactions with the \\ central nervous system through the vagus nerve, thoracolumbar connections \\ and sacral nerves, which provide opportunities for disease-modifying bioelectric \\ neuromodulation therapy. \\ - Sacral nerve stimulation (SNS) to treat faecal incontinence is the only \\ neuromodulation protocol for a gastrointestinal disorder that is currently in general \\ use; adapted SNS to selectively stimulate efferent pathways to treat constipation is \\ not in general use. \\ - Inhibition of gastrointestinal inflammation might be possible via vagal nerve \\ stimulation (VNS) or sympathetic nerve stimulation, and limited clinical testing \\ suggests effectiveness of cervical VNS. \\ - The vagus nerve carries signals for feeding, whose block might reduce appetite and \\ treat obesity; however, electrical block of vagal afferents had variable clinical success, \\ whereas direct stimulation of afferent endings at the gastric surface reduced satiety \\ in some studies. \\ - Gastric electrical stimulation stimulates afferent endings at the gastric surface, which, \\ in some studies, reduced postprandial nausea in patients with gastroparesis and \\ reduced weight gain in patients with obesity. \\ - Bioelectric neuromodulation might be a valuable treatment for several \\ gastrointestinal disorders but further investigations into the underlying mechanisms, \\ placement of stimulating electrodes, stimulus parameters and patient populations to \\ optimize effectiveness are still required.
}

Enteric nervous system (ENS). The nervous system embedded in the wall of the gastrointestinal tract, in the gallbladder and the pancreas.

Efferent

Refers to nerve pathways from the CNS and ENS to muscle, gland and epithelia.

Afferent

Refers to nerve pathways that carry sensory information from tissues to the CNS and ENS.

Sympathetic postganglionic neurons

Neurons of sympathetic pathways whose cell bodies reside in ganglia outside the CNS and ENS

Cervical sympathetic chain The part of the chain of sympathetic ganglia in the neck.

\section{Ganglia}

Collections of the cell bodies of autonomic neurons.

C fibres

Axons that conduct at low speeds (approximately $1 \mathrm{~m} / \mathrm{s}$ ).

Enteroendocrine cells Endocrine cells that are found in the lining of the gastrointestinal tract. including its physical state (for example, gastric fullness, muscle activity, temperature or gastric acidity), properties of its contents (for example, nutrient chemistry or osmolarity) and challenges to homeostasis (for example, inflammation, ischaemia or presence of toxins or pathogenic bacteria) ${ }^{6}$. The sensory innervation provides information that initiates feedback control to the digestive organs and behavioural changes (for example, feeding or expulsion of toxins) and causes conscious sensations such as pain, nausea, hunger or satiety. Furthermore, sensory neurons release mediators from their peripheral ends, providing efferent function. Because of their important and potentially disease-modifying roles, the vagus nerve, thoracolumbar nerves and sacral nerves have all been targeted with bioelectric neuromodulation therapies that aim to modify gut function in a reversible and and electrical current (TABLE 1).

\section{Vagal pathways to the digestive organs}

The vagus nerve consists of afferent nerve fibres (the majority of vagal axons) that project from visceral organs to the lower brainstem and efferent nerve fibres from the lower brainstem that connect with the viscera ${ }^{7}$. Axons of some sympathetic postganglionic neurons that originate from the cervical sympathetic chain and innervate the gastrointestinal organs also run in the vagus. They can be observed by catecholamine fluorescence or by tyrosine hydroxylase immunoreactivity, including in the human vagus $^{8,9}$. Denervation experiments in cats show that the majority of sympathetic axons in the vagus come from cervical sympathetic chain ganglia ${ }^{10}$. The vagus nerve supplies afferent and efferent fibres to all organs concerned with digestion: the oesophagus, stomach, small and large intestines, liver, pancreas and gallbladder. The vagus also supplies other organs, notably the heart, lungs and larynx. These additional connections are important highly controllable manner using implanted electrodes to consider because they can be inadvertently affected by stimuli that are directed at gastrointestinal afferent or efferent pathways.

Vagal afferent neurons. The afferent neurons of the vagus nerve have cell bodies in the nodose and jugular ganglia, and central projections that synapse in the nucleus tractus solitarius. They give rise to unmyelinated, slow-conducting axons (C fibres) ${ }^{7}$. Three types of vagal afferent fibre endings in the tubular gastrointestinal tract can be distinguished by their morphologies and targets: mucosal free endings, intraganglionic laminar endings and intramuscular arrays ${ }^{11}$. The mucosal endings form dense plexuses close to the lining epithelium and receive local hormonal signals from closely apposed basal surfaces of enteroendocrine cells ${ }^{12,13}$. Hormones that signal via vagal afferent neurons include cholecystokinin, 5-hydroxytryptamine, ghrelin, glucagon-like peptide 1 (GLP-1) and others. The enteroendocrine cells that release these hormones detect nutrients, and modulation of appetite (feeding behaviour) is one of the effects of this signalling. The intraganglionic laminar endings and intramuscular arrays are mechanosensitive and signal, for example, gastric fullness ${ }^{6,11}$.

Vagal efferent pathways. The majority of efferent axons in the vagus come from brainstem nuclei: the nucleus ambiguus and the dorsal motor nucleus of the vagus ${ }^{7}$. Neurons in the nucleus ambiguus provide direct innervation of the striated muscle of the oesophagus, although many have collaterals that innervate enteric neurons ${ }^{14}$. These neurons control oesophageal peristaltic movements ${ }^{15}$. Neurons in the dorsal motor nucleus are pre-enteric: they innervate enteric ganglia in the stomach, small and large intestines, biliary tract and pancreas. These neurons control gastrointestinal and gall bladder motility, gastric acid secretion and pancreatic enzyme release $^{16}$. The sympathetic pathways that reach the gut via the vagus are similar to the thoracolumbar pathways.

\section{Thoracolumbar pathways}

The thoracolumbar nerves carry afferent axons from the gastrointestinal tract that respond to painful stimuli and nociceptive events ${ }^{17}$ and efferent (sympathetic) pathways. The well-established sympathetic efferent pathways are those that inhibit gastrointestinal transit (by inhibiting movements of non-sphincter regions and by contracting (closing) sphincters), vasoconstrictor pathways and secretomotor inhibitory pathways ${ }^{15}$. However, sympathetic pathways that have anti-inflammatory effects have now also been discovered and are discussed later.

\section{Sacral nerves and pelvic nerve pathways}

The sacral nerves contain both autonomic and somatic efferents that control functions of the colon and rectum ${ }^{18}$. Autonomic fibres run in the pelvic nerves and influence motility and blood flow in the colon and rectum. A small number of sympathetic axons also run in the pelvic nerves. Somatic efferents in the pudendal nerves contract the external anal sphincter. Afferent fibres that join the pelvic nerves provide sensation from the anal canal and detect the physiological states of the colon and rectum ${ }^{11}$. 
Autonomic and somatic

efferents

Efferent neurons that belong to the autonomic nervous system and efferent neurons that are involved in somatic (skeletal muscle) control.

Superantigens Antigens that cause nonspecific activation of T cells resulting in polyclonal $\mathrm{T}$ cell activation and massive cytokine release.

Oxidative burst The rapid release of reactive oxygen species (superoxide radicals and hydrogen peroxide)

\section{Nerve stimulation for IBD}

\section{Epidemiology and current treatments}

Crohn's disease and ulcerative colitis, collectively termed IBD, are the most common immune-mediated disorders of the gastrointestinal $\operatorname{tract}^{19}$. They are debilitating, costly conditions whose incidences are highest in developed countries, with up to 5 million patients affected ${ }^{19}$. Newly developing countries in Asia, South America and the Middle East have relatively low disease incidences, but have some of the highest rates of increase $e^{20,21}$.

IBD often manifests in young adulthood and progressively worsens over a patient's lifetime $e^{19,22,23}$. The exact pathologic causes of IBD remain elusive, but genetic susceptibility, hazards to gut health (for example, diet, stress, appendectomy and/or excessive hygiene) and reduced diversity in intestinal microbiota are contributors to disease development and progression ${ }^{19}$. The mechanisms generating inflammation in IBD are thought to involve an inappropriate or exaggerated response to gut micro$\operatorname{organisms}^{24}$. Microbial superantigens that can produce a so-called cytokine storm (massive release of cytokines), which effectively turns off the immune response by

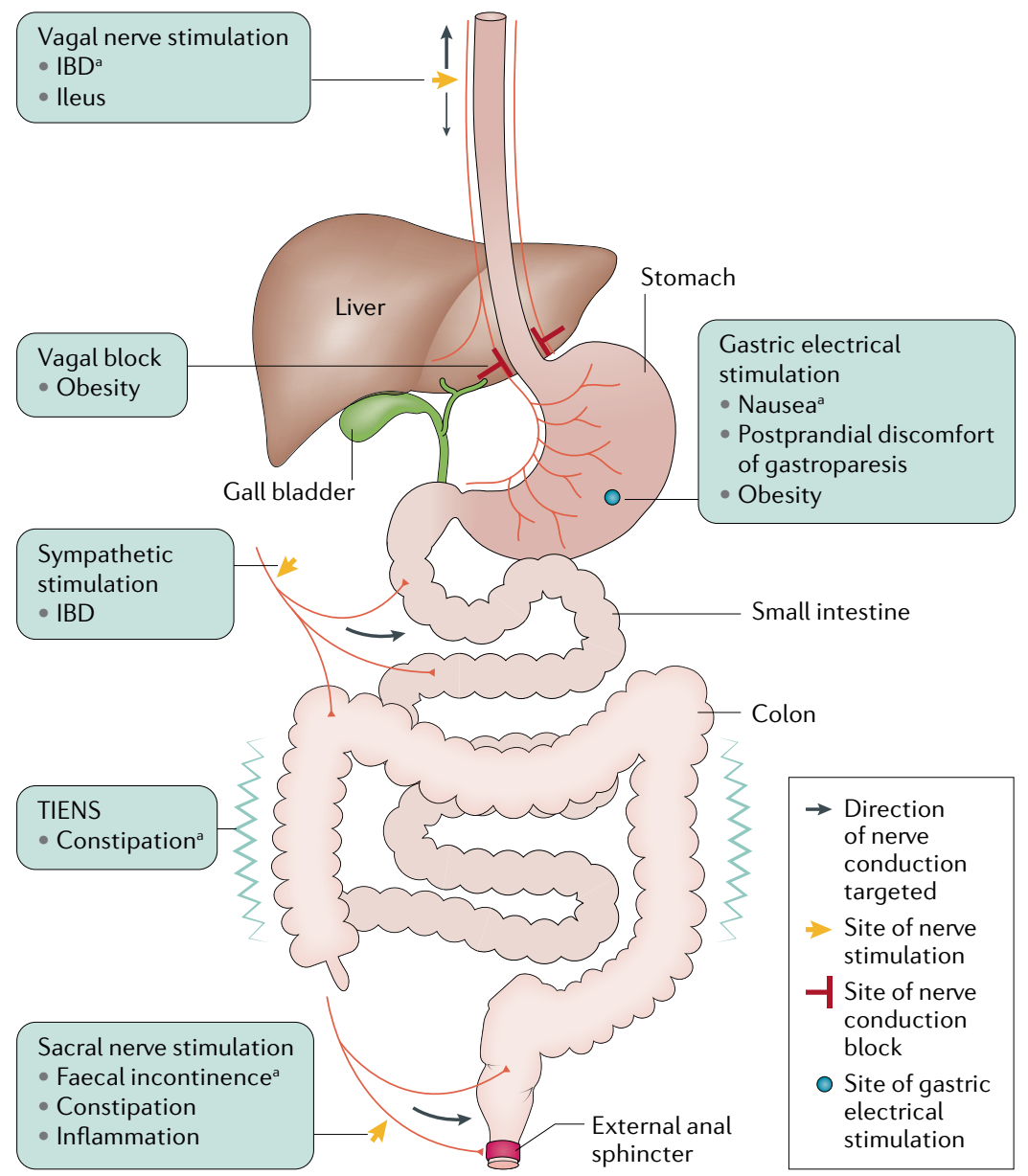

Fig. 1 | Sites of bioelectric neuromodulation to change gastrointestinal functions. Neuromodulation has been applied at several sites on nerves supplying the gastrointestinal tract in animal studies and experimental clinical settings for the treatment of multiple gastrointestinal disorders. For transcutaneous interferential electrical nerve stimulation (TIENS), high-frequency pulse trains are applied to both sides of the body. ${ }^{a}$ Conditions with evidence or potential of effectiveness of bioelectric neuromodulation in humans, based on clinical investigation rather than extrapolation from animal studies. overactivation, have been proposed to have a role, particularly in the acute severe form of the disease ${ }^{24,25}$. Macrophage-derived cytokines, such as TNF, IL-6 and IL-1, are among the first mediators to be released after an inflammatory challenge, causing a cascade of inflammatory actions, including the recruitment of other leukocyte populations (neutrophils, eosinophils, $\mathrm{T}$ cells and mast cells), granule exocytosis, production and activation of metalloproteinases and induction of the oxidative burst ${ }^{24}$. Cytokines drive intestinal inflamm czation, tissue damage and downstream complications, such as intestinal stenosis, rectal bleeding, abscess and fistula formation ${ }^{26}$ and even the development of extraintestinal manifestations, such as arthritis ${ }^{27}$.

Current treatments of IBD comprise anti-inflammatory and immunosuppressive agents and include thiopurines, which inhibit clonal expansion of lymphocytes, steroidal and nonsteroidal anti-inflammatory drugs and biological therapies (such as anti-TNF and anti-a4-integrin agents). Antibiotics are also used to decrease intestinal bacterial load ${ }^{28}$. Major drawbacks of these treatments include adverse effects, such as systemic immunosuppression, a lack of effectiveness in certain patients and development of refractory disease with continued treatment $\mathrm{t}^{29-31}$. Other serious safety concerns with the long-term use of biological therapies, such as anti-TNF, include development of opportunistic infections, reactivation of latent tuberculosis and development of haematological malignancies ${ }^{30}$. Despite the common use of expensive biological treatments for IBD, the disease becomes refractory in many patients with Crohn's disease and surgical resection of the inflamed gut is then the only treatment option. Furthermore, in a substantial proportion of these patients the disease recurs despite ongoing biological or pharmacological preventive treatment ${ }^{32}$. Thus, new therapies that are more effective and have fewer adverse effects than current treatments are needed.

\section{Neural control of gut inflammation}

Influence of vagal pathways on gut inflammation. The ability of vagal pathways to inhibit inflammation within the gut has been demonstrated in animal models ${ }^{33,34}$. The involved mechanisms are not yet fully understood and might differ with gut location and type of inflammatory stimulus used. Vagal stimulation can inhibit gut inflammation via different mechanisms, and which of these is most relevant to treating IBD in humans is yet to be determined but might differ depending on the site, cause and severity of the inflammation. The most widely accepted animal models of colitis involve luminal application of inflammatory agents such as dextran sodium sulfate (DSS) or 2,4,6-trinitrobenzenesulfonate (TNBS). Two studies have shown that electrical vagal stimulation can inhibit inflammation in such models ${ }^{33,34}$, but neither of these studies investigated which vagal neurons are involved in this effect. Another study showed that sub-diaphragmatic vagotomy increased inflammation in the gut following oral DSS or intracolonic dinitrobenzenesulfonate $e^{35}$. Denervation of the spleen had a similar effect, whereas splenectomy did not ${ }^{36}$. The authors concluded that vagal control of splenic cytokine release 


\section{Cholinergic agents \\ Drugs whose action relies on the neurotransmitter acetylcholine. \\ Pathogen-associated molecular patterns (PAMPs). Molecular motifs conserved within microorganisms that are recognized by cells of the innate immune system. \\ Capsaicin-sensitive Refers to neurons that are activated by the capsaicin compound from red peppers, which in high enough concentrations causes the neurons to degenerate}

Myenteric plexus A plexus of nerves and ganglia of the enteric nervous system, located within the external muscle of the gastrointestina tract.

Tyrosine-hydroxylaseimmunoreactive nerve endings

The endings of neurons that contain the enzyme tyrosine hydroxylase, which is necessary for the synthesis of adrenaline, dopamine and noradrenaline. suppresses inflammation in the intestine. Another group showed that cutting the coeliac branches of the vagus that supply the intestine (selective intestinal denervation) did not aggravate colitis ${ }^{37}$. Stimulation of vagal pathways by central-acting cholinergic agents was reported to inhibit inflammation in DSS-induced colitis via a similar indirect mechanism dependent on the integrity of both the vagus nerve and the innervation of the spleen ${ }^{36}$. These studies discounted any involvement of direct vagal pathways to the gut in these experimental conditions, but do not preclude that vagal pathways might also inhibit gastrointestinal inflammation by an action directly in the intestine, as is seen in ileus (discussed later).

Suppression of intestinal inflammation possibly involves a mechanism similar to that observed to inhibit systemic inflammation, namely the vagovagal cholinergic anti-inflammatory reflex ${ }^{38,39}$. This reflex is initiated through the actions of cytokines and pathogen-associated molecular patterns (PAMPs) on vagal sensory nerve endings. The vagal afferents synapse in the brainstem to stimulate vagal efferent pathways that indirectly influence the spleen, reducing the production and release of circulating cytokines and diminishing adaptive immune responses ${ }^{38,39}$. This theory has been challenged and sympathetic pathways have been suggested to carry the efferent arm of the anti-inflammatory reflex $^{40,41}$. Currently available evidence does not adequately resolve whether vagal efferents, sympathetic efferents or both are involved in the reflex control of intestinal inflammation.

Capsaicin-sensitive afferents and inflammation regulation. Both electrical and physiological stimulation of afferent nerves, including afferent nerves to the digestive tract, causes release of transmitters, the most pertinent being tachykinins and calcitonin-generelated peptide (CGRP), from their peripheral ends ${ }^{42}$. Moreover, capsaicin-sensitive afferent fibres exert protective anti-inflammatory effects, reducing mucosal damage in the gastrointestinal tract via peptide release from their peripheral endings ${ }^{43-46}$. Rat and mouse models of gastric ulcer show that sensory afferents innervating the stomach release CGRP to reduce mucosal

Box 1 Challenges for bioelectric therapies
- Achieving a beneficial, clinically significant therapeutic
end point.
- Achieving disease-modifying rather than symptomatic
relief.
- Defining an adequate nonstimulated control with
which to compare.
- Safety.
- Optimizing stimulation parameters.
- Optimizing stimulation location.
- Monitoring effectiveness of stimulation at the
stimulation site.
- Off-target effects.
- Maintenance of effectiveness over time.
- Inter-patient variability.
- Achieving feed-back (closed-loop) control.

damage and to promote mesenteric and mucosal blood flow $^{44,45}$. Following ablation of afferents through capsaicin application in rats, the numbers and sizes of gastric mucosal lesions in response to indomethacin, ethanol or cysteamine insult were significantly greater ${ }^{43}$. By contrast, the acute application of capsaicin in rats induced neuropeptide release and reduced the extent of ethanol-induced gastric lesions if used at the time of damage ${ }^{43,46}$. This effect is mediated by the release of CGRP from afferent nerve endings before they degenerate hours to days after capsaicin administration ${ }^{46}$. The acute capsaicin-induced gastric mucosal protection from ethanol injury in rats was inhibited by the CGRP receptor antagonist peptide CGRP 8-37 and a polyclonal anti-CGRP antiserum ${ }^{46}$.

The protective anti-inflammatory effects of afferents are also seen in rat and rabbit models of colitis. Following the degeneration of capsaicin-sensitive afferent fibres, the severity of TNBS-induced colitis was increased, but colonic transit time did not change, indicating that gut motility was not affected ${ }^{47-49}$. These effects are likely mediated by the involvement of the neuropeptide CGRP, as CGRP 8-37 exacerbated TNBS-induced mucosal damage in rats ${ }^{50}$. These studies do not distinguish between the source of afferent fibres as being from the vagus nerve or spinal nerves, both of which provide CGRP afferents to the gastrointestinal tract ${ }^{51,52}$.

Vagal efferent fibres. Perhaps the best evidence of a local intestinal anti-inflammatory effect of efferent vagal fibres comes from experiments in which inflammation was induced using mechanical stimuli applied to the outside of the intestines - a model of postoperative ileus that activates macrophages within the muscularis externa ${ }^{53,54}$. This pathway is independent of the vagal influences on the spleen, as splenic denervation does not prevent the anti-inflammatory effect, but does involve vagal stimulation of acetylcholine release from enteric neurons, which then acts on intestinal macrophages ${ }^{54}$. This mechanism might be relevant in advanced Crohn's disease in which relapse following surgery is strongly correlated with inflammation in the external layers of the gut wall around the myenteric plexus ${ }^{55}$.

Influence of sympathetic pathways on gut inflammation. Vagal influences on inflammation in the gut have only been discovered in the past 20 years, but a substantial body of evidence exists that shows that adrenergic nerves directly control inflammation in the gut ${ }^{56}$. Tyrosine-hydroxylase-immunoreactive nerve endings are found near resident macrophages in the lamina propria of the mucosa, in the submucosa, around the ganglia of the myenteric plexus and innervating lymphoid follicles (Peyer's patches) ${ }^{56}$. They do not, however, come in contact with the extensive network of macrophages in the muscle layers, and actions of sympathetic nerves on this subgroup of resident immune cells might be indirect via their demonstrated influences on enteric neurons $^{57,58}$, some of which innervate these macrophages ${ }^{54}$. Macrophages express various types of adrenergic receptors. $\beta$-Receptors mediate the anti-inflammatory actions of agonists on gut-derived macrophages in vitro ${ }^{59}$, as well 
a Afferent signals

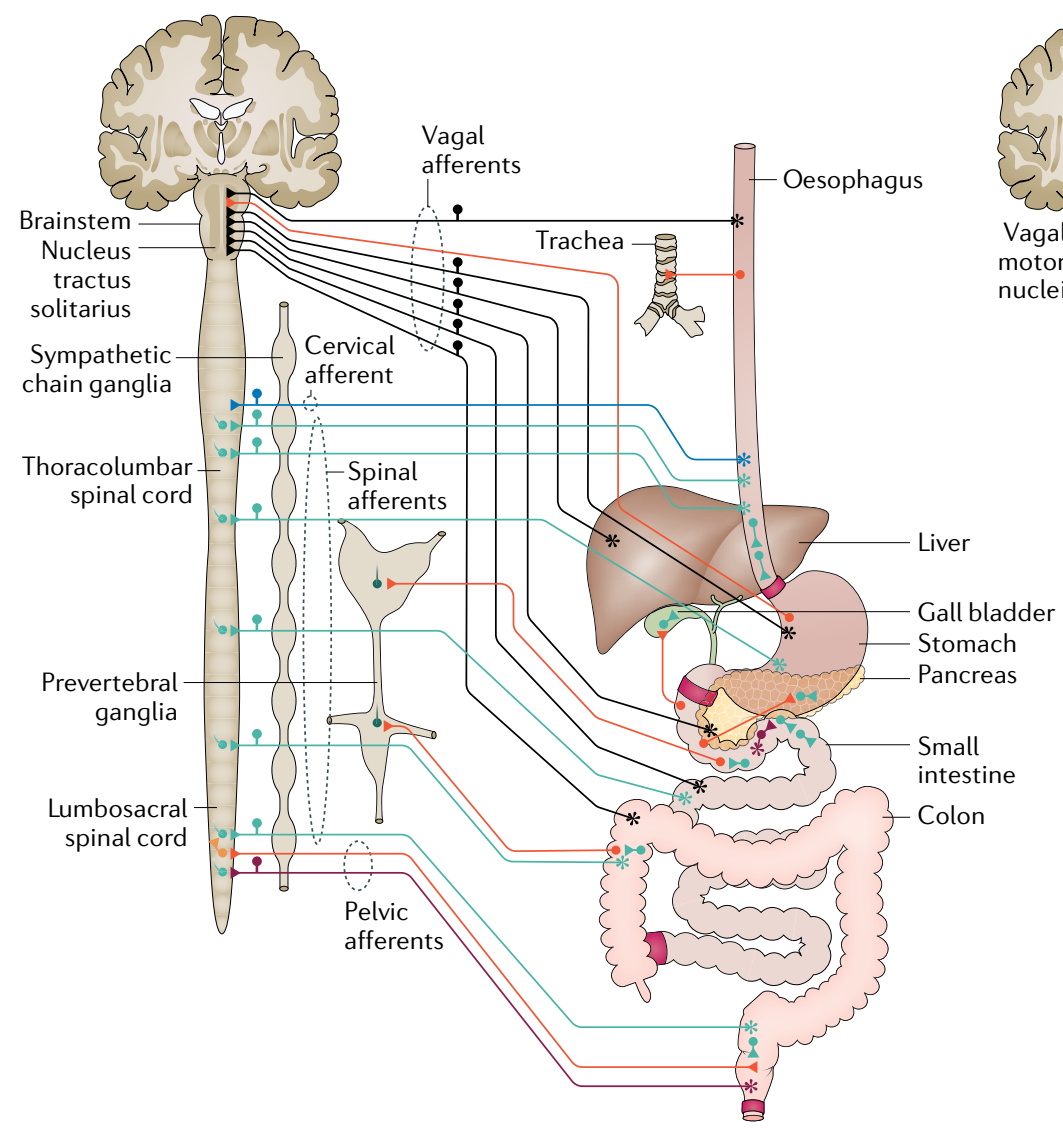

b Efferent signals

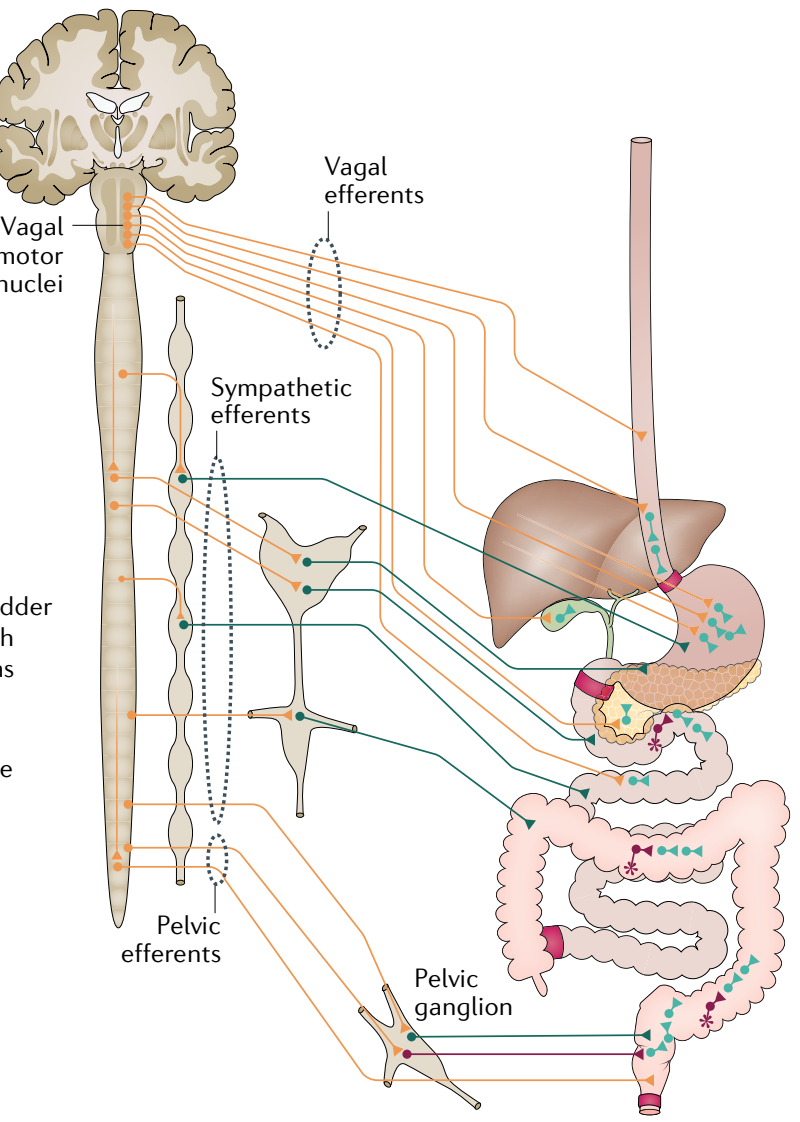

- Vagal afferent neuron

- Cervical afferent neuron

- Pre-enteric and preganglionic neuron
- Intestinofugal neuron

- Spinal afferent neuron

- Pelvic postganglionic neuron
- Sympathetic postganglionic neuron

Sphincter
- Enteric neuron

$\rightarrow$ Afferent neuron ending

^ Afferent neuron

Fig. 2 | The extrinsic innervation of the gastrointestinal tract. The neural connections between the central nervous system (CNS), sympathetic ganglia, the enteric nervous system (ENS) and gastrointestinal effector tissues are complex. a Afferent signals travel along connections from the digestive system to other organs and the CNS. Vagal afferents end in the nucleus tractus solitarius and spinal afferents end in the thoracolumbar spinal cord. In addition, cervical afferents connect the oesophagus to the cervical spinal cord. Some afferent pathways involve intestinofugal neurons that project away from the gut to the CNS, sympathetic ganglia, gallbladder, pancreas and trachea. Some sensory information is transferred by intrinsic sensory neurons to the ENS. $\mathbf{b}$ | Efferent signals travel along connections from the CNS to the digestive system. Pathways from the CNS reach the ENS and gastrointestinal effector tissues through vagal, sympathetic and pelvic pathways. The sympathetic pathways pass through sympathetic chain ganglia. The vagal efferent neurons have cell bodies in the dorsal motor nucleus of the vagus and nucleus ambiguus. Thoracolumbar and lumbosacral efferents have cell bodies in the autonomic intermediolateral nuclei and Onuf's nucleus (see FIC. 4). Neurons of vagal medullary and pelvic spinal outflows synapse with enteric neurons, and many gut-projecting sympathetic neurons with cell bodies in prevertebral ganglia are also pre-enteric neurons. Some neurons in sympathetic prevertebral ganglia receive both CNS and ENS inputs.

as in colitis models in vivo ${ }^{60}$. These anti-inflammatory effects are distinct from the motility-modifying effects of adrenergic nerves that occur via $\alpha$-receptors ${ }^{58}$. These disparate effects of sympathetic nerves complicate the interpretation of experiments using nonselective sympathectomy to study the regulation of gut inflammation. Local inflammation is well known to inhibit gut motility via activation of sympathetic nerves and sympathectomy can prevent inflammation-induced gut stasis ${ }^{61,62}$, which indirectly reduces inflammation because bacteria no longer accumulate in the small intestine when it is able to propel its contents. Despite this, careful experimentation shows that inflammation in experimental
IBD is inhibited by intermittent electrical stimulation of the sympathetic nerves to the gut, and it is known that intermittent electrical stimulation does not cause sustained inhibition of motility; therefore, the inhibition of inflammation is direct ${ }^{37}$.

Interestingly, a new study suggests that $\beta$-blockers exacerbate inflammation in Crohn's disease in humans, increasing the incidence of relapse following surgery ${ }^{63}$. An interpretation of these data is that sympathetic anti-inflammatory pathways are activated in Crohn's disease and that their effect is mediated via $\beta$-receptors. Taken together, these results suggest that sympathetic nerves to the gut do inhibit inflammation, but sustained 
Table 1 | Clinical studies of bioelectric neuromodulation for gastrointestinal disorders

\begin{tabular}{|c|c|c|c|c|c|c|}
\hline Disease & $\begin{array}{l}\text { Stimulation site } \\
\text { and method }\end{array}$ & $\begin{array}{l}\text { Electrode type } \\
\text { and/or device name }\end{array}$ & $\begin{array}{l}\text { Stimulation } \\
\text { parameters }\end{array}$ & $\begin{array}{l}\text { Stimulus } \\
\text { duration }\end{array}$ & Study design & Outcome \\
\hline \multicolumn{7}{|l|}{ IBD } \\
\hline $\begin{array}{l}\text { Ileo-caecal } \\
\text { Crohn's } \\
\text { disease }^{65}\end{array}$ & $\begin{array}{l}\text { - Unilateral } \\
\text { cervical vagus } \\
\text { - Stimulation }\end{array}$ & $\begin{array}{l}\text { - Bipolar, helical } \\
\text { - Model } 302 \\
\text { (Cyberonics) }\end{array}$ & $\begin{array}{l}0.5 \mathrm{~ms} ; 10 \mathrm{~Hz} \\
0.5-1.25 \mathrm{~mA}\end{array}$ & $\begin{array}{l}30 \mathrm{~s} \text { ON, } 5 \text { min } \\
\text { OFF; } 24 \text { h/day }\end{array}$ & Open-label & $\begin{array}{l}\text { Clinical, biological, } \\
\text { endoscopic remission } \\
\text { in } 5 \text { of } 7 \text { patients }\end{array}$ \\
\hline $\begin{array}{l}\text { Ulcerative } \\
\text { proctitis }^{68}\end{array}$ & $\begin{array}{l}\text { - Sacral nerve } \\
\text { - Stimulation }\end{array}$ & $\begin{array}{l}\text { - Multi-ring electrode } \\
\text { array } \\
\text { - InterStim II } \\
\text { neuromodulator } \\
\text { (Medtronic) }\end{array}$ & $\begin{array}{l}210 \mathrm{~ms} ; 14 \mathrm{~Hz} \\
0.5-1.5 \mathrm{~V}\end{array}$ & Not disclosed & Case study & $\begin{array}{l}\text { Decrease in faecal } \\
\text { incontinence and disease } \\
\text { activity scores }(n=1)\end{array}$ \\
\hline \multicolumn{7}{|c|}{ Post-operative ileus } \\
\hline $\begin{array}{l}\text { Surgery-induced } \\
\text { postoperative } \\
\text { ileus }^{71}\end{array}$ & $\begin{array}{l}\text { - Unilateral } \\
\text { posterior } \\
\text { abdominal vagus } \\
\text { - Stimulation }\end{array}$ & $\begin{array}{l}\text { - S-Cuff, bipolar } \\
\text { Delta electrode } \\
\text { (Inomed) }\end{array}$ & $\begin{array}{l}0.5,1 \text { or } 2 \mathrm{~ms} \\
20 \mathrm{~Hz} ; 2.5 \mathrm{~mA}\end{array}$ & $\begin{array}{l}2 \mathrm{~min} \text { each } \\
\text { before and after } \\
\text { surgery }\end{array}$ & $\begin{array}{l}\text { Randomized, } \\
\text { double-blind, } \\
\text { placebo- } \\
\text { controlled }\end{array}$ & $\begin{array}{l}\text { Reduction in } \\
\text { pro-inflammatory } \\
\text { cytokine production versus } \\
\text { sham }(n=7 \text { versus } n=5 ; \\
P<0.05)\end{array}$ \\
\hline \multicolumn{7}{|l|}{ Obesity } \\
\hline Morbid obesity ${ }^{88}$ & $\begin{array}{l}\text { - Bilateral } \\
\text { abdominal vagus } \\
\text { - Blocking }\end{array}$ & $\begin{array}{l}\text { - Bipolar, cuff } \\
\text { - vBloc } \\
\text { (Enteromedics) }\end{array}$ & $\begin{array}{l}5,000 \mathrm{~Hz} \\
1-6 \mathrm{~mA}\end{array}$ & $\begin{array}{l}5 \mathrm{~min} \text { ON, } 5 \text { min } \\
\text { OFF; } 12 \text { h/day }\end{array}$ & $\begin{array}{l}\text { Open-label, no } \\
\text { placebo }\end{array}$ & $\begin{array}{l}\text { Safety study (good profile); } \\
\text { EWL of }>25 \% \text { at } 6 \text { months } \\
(n=31 ; P<0.001)\end{array}$ \\
\hline Morbid obesity ${ }^{90}$ & $\begin{array}{l}\text { - Bilateral } \\
\text { abdominal vagus } \\
\text { - Blocking }\end{array}$ & $\begin{array}{l}\text { - Bipolar, cuff } \\
\text { vBloc } \\
\text { (Enteromedics) }\end{array}$ & $3-8 \mathrm{~mA}$ & $\begin{array}{l}5 \mathrm{~min} \text { ON, } 5 \text { min } \\
\text { OFF; } 9-16 \text { h/day }\end{array}$ & $\begin{array}{l}\text { Randomized, } \\
\text { prospective, } \\
\text { double-blind, } \\
\text { multicentre, } \\
\text { no placebo }\end{array}$ & $\begin{array}{l}\text { No differences in weight } \\
\text { loss versus sham }(n=192 \text {, } \\
17 \pm 2 \% \text { EWL versus } n=102 \\
16 \pm 2 \% \text { EWL) }\end{array}$ \\
\hline $\begin{array}{l}\text { Morbid obesity } \\
\text { and one } \\
\text { co-morbidity }\end{array}$ & $\begin{array}{l}\text { - Bilateral } \\
\text { abdominal vagus } \\
\text { - Blocking }\end{array}$ & $\begin{array}{l}\text { - Bipolar, cuff } \\
\text { vBloc } \\
\text { (Enteromedics) }\end{array}$ & $6-8 \mathrm{~mA}$ & $\begin{array}{l}\text { Not disclosed; } \\
\text { Up to } 12 \mathrm{~h} / \text { day }\end{array}$ & $\begin{array}{l}\text { Randomized, } \\
\text { double-blind, } \\
\text { placebo- } \\
\text { controlled }\end{array}$ & $\begin{array}{l}\text { Increased weight loss } \\
\text { versus sham }(n=162,24 \% \\
\text { versus } n=77,16 \% \text { EWL; } \\
P=0.002) ; \text { primary efficacy } \\
\text { objective not reached } \\
(P=0.71)\end{array}$ \\
\hline Morbid obesity ${ }^{91}$ & $\begin{array}{l}\text { - Bilateral } \\
\text { abdominal vagus } \\
\text { - Blocking }\end{array}$ & $\begin{array}{l}\text { - Bipolar, cuff } \\
\text { - vBloc } \\
\text { (Enteromedics) }\end{array}$ & $6 \mathrm{~mA}$ & $\begin{array}{l}\text { Not disclosed; } \\
\geq 12 \mathrm{~h} / \text { day }\end{array}$ & $\begin{array}{l}\text { Randomized, } \\
\text { double-blind, } \\
\text { placebo- } \\
\text { controlled }\end{array}$ & $\begin{array}{l}\text { Increased weight loss } \\
\text { versus sham } \\
\text { at } 18 \text { months }(n=142,23 \% \\
\text { EWL versus } n=64,10 \% \\
\text { EWL; } P<0.0001)\end{array}$ \\
\hline $\begin{array}{l}\text { Depression and } \\
\text { obesity }^{101}\end{array}$ & $\begin{array}{l}\text { - Unilateral } \\
\text { cervical vagus } \\
\text { - Stimulation }\end{array}$ & $\begin{array}{l}\text { - Bipolar, helical } \\
\text { - NCP Model } 101 \\
\text { (Cyberonics) }\end{array}$ & $\begin{array}{l}0.5 \mathrm{~ms} ; 30 \mathrm{~Hz} \\
0.25-1.5 \mathrm{~mA}\end{array}$ & $\begin{array}{l}30 \mathrm{~s} \text { ON, } 5 \text { min } \\
\text { OFF; continuous } \\
\text { cycle }\end{array}$ & $\begin{array}{l}\text { Retrospective } \\
\text { clinical } \\
\text { observation, } \\
\text { no placebo }\end{array}$ & $\begin{array}{l}\text { Correlation between } \\
\text { decrease in BMl and } \\
\text { stimulation duration } \\
(n=14 ; \text { Pearson } r=0.73 \text {, } \\
P<0.003)\end{array}$ \\
\hline Obesity $^{112}$ & $\begin{array}{l}\text { - Anterior gastric } \\
\text { wall at the lesser } \\
\text { curvature } \\
\text { GES }\end{array}$ & $\begin{array}{l}\text { - Bipolar, implantable } \\
\text { gastric stimulator } \\
\text { - Transcend } \\
\text { (Transneuronix) }\end{array}$ & $\begin{array}{l}0.208 \mathrm{~ms} \\
40 \mathrm{~Hz} ; 10 \mathrm{~mA}\end{array}$ & $\begin{array}{l}\text { 2s ON, 3s OFF; } \\
\text { not disclosed }\end{array}$ & Open-label & $\begin{array}{l}\text { Safety study (good } \\
\text { profile). Men }(n=5) \\
\text { lost } 25 \% \text { EWL but } \\
\text { women }(n=7) \text { gained } \\
6.2 \% \text { EWL versus } \\
\text { pre-surgery weight } \\
(P<0.001)\end{array}$ \\
\hline Obesity $^{113}$ & $\begin{array}{l}\text { - Anterior gastric } \\
\text { wall at the lesser } \\
\text { curvature } \\
\text { - GES }\end{array}$ & $\begin{array}{l}\text { - Bipolar } \\
\text { - Design and } \\
\text { manufacturer not } \\
\text { disclosed }\end{array}$ & Not disclosed & Not disclosed & Pilot open-label & $\begin{array}{l}24 \% \text { mean EWL versus } \\
\text { presurgery weight at } \\
36 \text { months follow-up point } \\
(n=10)\end{array}$ \\
\hline Obesity $^{106}$ & $\begin{array}{l}\text { - Anterior gastric } \\
\text { wall at the lesser } \\
\text { curvature } \\
\text { GES }\end{array}$ & $\begin{array}{l}\text { - Bipolar } \\
\text { - Transcend II } \\
\text { (Medtronic) }\end{array}$ & $\begin{array}{l}0.208 \mathrm{~ms} \\
40 \mathrm{~Hz} \\
\text { (individually } \\
\text { set) }\end{array}$ & $\begin{array}{l}\text { 2s ON, 3s OFF; } \\
\text { not disclosed }\end{array}$ & $\begin{array}{l}\text { Randomized, } \\
\text { prospective, } \\
\text { double-blind, } \\
\text { multicentre, } \\
\text { placebo- } \\
\text { controlled }\end{array}$ & $\begin{array}{l}\text { No difference versus } \\
\text { control group }(n=9,611 \\
11.8 \% \pm 17.6 \% \text { EWL versus } \\
n=94,7 \% \pm 16.9 \% \text { EWL; } \\
P=0.717)\end{array}$ \\
\hline $\begin{array}{l}\text { Overweight and } \\
\text { type } 2 \text { diabetes } \\
\text { mellitus }^{107}\end{array}$ & $\begin{array}{l}\text { - Anterior and } \\
\text { posterior antral } \\
\text { areas } \\
\text { GES }\end{array}$ & $\begin{array}{l}\text { - Three bipolar pairs } \\
\text { - Tantalus-DIAMOND } \\
\text { (Metacure) }\end{array}$ & Not disclosed & $\begin{array}{l}\text { Not disclosed; } \\
\text { closed loop } \\
\text { (electrodes in } \\
\text { fundus detect } \\
\text { gastric filling) }\end{array}$ & Open-label & $\begin{array}{l}\text { No weight loss ( } n=22 \text {; } \\
P>0.05)\end{array}$ \\
\hline
\end{tabular}


Table 1 (cont.) | Clinical studies of bioelectric neuromodulation for gastrointestinal disorders

\begin{tabular}{|c|c|c|c|c|c|c|}
\hline Disease & $\begin{array}{l}\text { Stimulation site } \\
\text { and method }\end{array}$ & $\begin{array}{l}\text { Electrode type } \\
\text { and/or device name }\end{array}$ & $\begin{array}{l}\text { Stimulation } \\
\text { parameters }\end{array}$ & $\begin{array}{l}\text { Stimulus } \\
\text { duration }\end{array}$ & Study design & Outcome \\
\hline \multicolumn{7}{|l|}{ Obesity (cont.) } \\
\hline Obesity $^{105}$ & $\begin{array}{l}\text { - Food sensor in } \\
\text { fundus; electrode } \\
\text { on the lesser } \\
\text { curvature (over } \\
\text { vagal branches) } \\
\text { - GES }\end{array}$ & $\begin{array}{l}\text { - Two bipolar pairs } \\
\text { - abiliti system } \\
\text { (IntraPace) }\end{array}$ & Not disclosed & $\begin{array}{l}\text { Not disclosed; } \\
\text { closed loop } \\
\text { (electrodes in } \\
\text { fundus detect } \\
\text { gastric filling) }\end{array}$ & Open-label & $\begin{array}{l}\text { EWL of combined cohort } \\
49.3 \pm 19.2 \% \text { at } 12 \text { months } \\
\text { ( } n=27), \text { EWL higher for } \\
\text { low BMl group ( } n=4 \text {, } \\
59.1 \pm 19.5 \% \text { ) than high BMI } \\
\text { group }(n=9,46.7 \pm 13.4 \% \text {; } \\
P<0.01)\end{array}$ \\
\hline Obesity $^{108}$ & $\begin{array}{l}\text { Trans-gastric } \\
\text { food-detecting } \\
\text { sensor in fundus, } \\
\text { electrode on the } \\
\text { lesser curvature } \\
\text { (over vagal } \\
\text { branches) } \\
\text { GES }\end{array}$ & $\begin{array}{l}\text { - Two bipolar pairs } \\
\text { - abiliti system } \\
\text { (IntraPace) }\end{array}$ & $\begin{array}{l}0.1-2 \mathrm{~ms} \\
\text { pulse train; } \\
40-120 \mathrm{~Hz} \\
4-30 \mathrm{~mA}\end{array}$ & $\begin{array}{l}\text { Not disclosed; } \\
\text { closed loop } \\
\text { (electrodes in } \\
\text { fundus detect } \\
\text { gastric filling) }\end{array}$ & $\begin{array}{l}\text { Prospective, } \\
\text { multicentre, } \\
\text { no placebo }\end{array}$ & $\begin{array}{l}27.5 \% \text { EWL at } \\
27 \text { months }(n=16)\end{array}$ \\
\hline \multicolumn{7}{|c|}{ Nausea and gastroparesis } \\
\hline Gastroparesis $^{121}$ & $\begin{array}{l}\text { - Into serosal } \\
\text { surface of } \\
\text { stomach } \\
\text {-GES }\end{array}$ & $\begin{array}{l}\text { - Four pairs of cardiac } \\
\text { pacing wires } \\
\text { - Enterra therapy } \\
\text { system (Medtronic) }\end{array}$ & $\begin{array}{l}300 \mathrm{~ms} ; 4 \mathrm{~mA} \\
10 \% \text { higher } \\
\text { frequency } \\
\text { than gastric } \\
\text { slow wave }\end{array}$ & $\begin{array}{l}\text { Not disclosed; } \\
\text { up to } 1 \mathrm{~h} \text { before } \\
\text { and } 3 \mathrm{~h} \text { after the } \\
\text { meal }\end{array}$ & Pilot, open-label & $\begin{array}{l}\text { Substantial reduction of } \\
\text { gastroparesis symptoms } \\
\text { after } 1 \text { month treatment } \\
(n=9 ; P=0.04)\end{array}$ \\
\hline $\begin{array}{l}\text { Diabetic } \\
\text { gastroparesis }\end{array}$ & $\begin{array}{l}\text { - Muscle wall } \\
\text { of the greater } \\
\text { curvature } \\
\text { - GES }\end{array}$ & $\begin{array}{l}\text { - Two intramuscular } \\
\text { - Enterra therapy } \\
\text { system (Medtronic) }\end{array}$ & $\begin{array}{l}0.33 \mathrm{~ms} \\
14 \mathrm{~Hz} ; 5 \mathrm{~mA}\end{array}$ & $0.1 \mathrm{~s}$ ON, $5 \mathrm{~s}$ OFF & $\begin{array}{l}\text { Double-blind, } \\
\text { cross-over, } \\
\text { prospective, } \\
\text { multicentre, } \\
\text { no placebo }\end{array}$ & $\begin{array}{l}57 \% \text { decrease of weekly } \\
\text { vomiting frequency versus } \\
\text { presurgery baseline }(n=55)\end{array}$ \\
\hline Gastroparesis $^{162}$ & $\begin{array}{l}\text { - Endoscopically } \\
\text { placed electrode } \\
\text { into the antrum } \\
\text { - Temporary GES }\end{array}$ & $\begin{array}{l}\text { - Bipolar, two } \\
\text { intramuscular } \\
\text { - Model no. } 4300 \\
\text { (Medtronic) }\end{array}$ & $\begin{array}{l}0.33 \mathrm{~ms} ; \\
14 \mathrm{~Hz} ; 5 \mathrm{~mA}\end{array}$ & $\begin{array}{l}0.1 \mathrm{~s} \mathrm{ON}, 5 \mathrm{~s} \text { OFF; } \\
72 \mathrm{~h} \text { continuous }\end{array}$ & $\begin{array}{l}\text { Randomized, } \\
\text { double-blind, } \\
\text { cross-over, } \\
\text { placebo- } \\
\text { controlled }\end{array}$ & $\begin{array}{l}\text { No change in average } \\
\text { vomiting scores } \\
\text { during stimulation } \\
\text { versus nonstimulation } \\
\text { (pooled data } n=58 ; \\
P=0.116 \text { ) }\end{array}$ \\
\hline \multicolumn{7}{|c|}{ Colorectal dysfunction } \\
\hline $\begin{array}{l}\text { Faecal } \\
\text { incontinence }^{145}\end{array}$ & $\begin{array}{l}\text { - Sacral spinal } \\
\text { nerves } \\
\text { - Stimulation }\end{array}$ & $\begin{array}{l}\text { - Foramen electrode } \\
\text { - Quadlead } 3886 \\
\text { (Medtronic) }\end{array}$ & $\begin{array}{l}0.21 \mathrm{~ms} ; \\
15 \mathrm{~Hz} \text {; current } \\
\text { adapted } \\
\text { to patient } \\
\text { tolerance }\end{array}$ & $5 \mathrm{~s}$ ON, $1 \mathrm{~s}$ OFF & Pilot, open-label & $\begin{array}{l}\text { Full continence in } 2 \text { of } \\
3 \text { patients; one patient } \\
\text { improved from gross } \\
\text { incontinence to minor } \\
\text { soiling }\end{array}$ \\
\hline $\begin{array}{l}\text { Faecal } \\
\text { incontinence }^{147}\end{array}$ & $\begin{array}{l}\text { - Sacral spinal } \\
\text { nerves } \\
\text { - Stimulation }\end{array}$ & $\begin{array}{l}\text { - Foramen electrode } \\
\text { - Quadripolar } \\
\text { (Medtronic) }\end{array}$ & $\begin{array}{l}0.21 \mathrm{~ms} ; \\
19 \mathrm{~Hz} \text {; current } \\
\text { adapted } \\
\text { to patient } \\
\text { tolerance }\end{array}$ & $20 \mathrm{~s}$ ON, $8 \mathrm{~s}$ OFF & $\begin{array}{l}\text { Randomized, } \\
\text { prospective, } \\
\text { open-label }\end{array}$ & $\begin{array}{l}\text { Reduction in mean } \\
\text { incontinence episodes } \\
\text { per week versus } \\
\text { non-implanted (best } \\
\text { supportive therapy) } \\
\text { controls }(n=60 \text { and } n=60 \text {; } \\
P<0.0001 \text { ) }\end{array}$ \\
\hline Constipation $^{151}$ & $\begin{array}{l}\text { - Anterior sacral } \\
\text { nerve } \\
\text { - Stimulation }\end{array}$ & $\begin{array}{l}\text { - Bipolar } \\
\text { - Type LBS } 53051 \\
\text { (Medelec) }\end{array}$ & $0.1 \mathrm{~ms} ; 2 \mathrm{~Hz}$ & Not disclosed & Pilot, open-label & $\begin{array}{l}\text { Differential effects on } \\
\text { colorectal motor activity } \\
\text { (depending on spinal level; } \\
n=5 \text { ) }\end{array}$ \\
\hline Constipation $^{153}$ & $\begin{array}{l}\text { - Anterior sacral } \\
\text { nerve } \\
\text { - Stimulation }\end{array}$ & Not disclosed & Not disclosed & Not disclosed & Open-label & $\begin{array}{l}\text { Decline in faecal } \\
\text { dysfunction score versus } \\
\text { baseline }(P<0.0001)\end{array}$ \\
\hline Constipation $^{158}$ & $\begin{array}{l}\text { - Transcutaneous } \\
\text { - Electrical } \\
\text { stimulation }\end{array}$ & Not disclosed & $80-150 \mathrm{~Hz}$ & $\begin{array}{l}30 \mathrm{~min} / \text { day for } \\
2 \text { months }\end{array}$ & $\begin{array}{l}\text { Randomized, } \\
\text { open-label }\end{array}$ & $\begin{array}{l}\text { Improved soiling } \\
\text { continence score in } 81 \% \\
\text { of patients ( } n=17 \text { of } 21 ; \\
P=0.0002 \text { ) }\end{array}$ \\
\hline Constipation $^{159}$ & $\begin{array}{l}\text { - Paraspinal and } \\
\text { abdominal } \\
\text { transcutaneous } \\
\text { Electrical } \\
\text { stimulation }\end{array}$ & $\begin{array}{l}\text { - Two paraspinal and } \\
\text { two abdominal } \\
\text { self-adhesive } \\
\text { conducting } \\
\text { - Vectorsurge } 5 \text { VS470 } \\
\text { (Metron Medical) }\end{array}$ & $\begin{array}{l}80-150 \mathrm{~Hz} \\
<40 \mathrm{~mA}\end{array}$ & $\begin{array}{l}\text { 20-min sessions; } \\
3 \text { times per week } \\
\text { for } 4 \text { weeks }\end{array}$ & $\begin{array}{l}\text { Randomized, } \\
\text { open-label }\end{array}$ & $\begin{array}{l}\text { Increased frequency of } \\
\text { colonic movements versus } \\
\text { pre-stimulation }(n=7 ; \\
P=0.008)\end{array}$ \\
\hline
\end{tabular}

EWL, excess weight loss; GES gastric electrical stimulation. 
stimulation of these nerves should be avoided, as it would cause stasis and exacerbate bacterial overgrowth and gut dysfunction ${ }^{64}$.

\section{VNS for IBD}

Experimental and clinical trials of vagal nerve stimulation (VNS) to alleviate IBD have had variable outcomes. The anti-inflammatory effects of VNS have been demonstrated in rodent models of colitis (TABLE 1). VNS (3 hours per day) applied to awake rats with TNBS-induced colitis effectively reduced weight loss, colonic histological score and molecular expression of pro-inflammatory cytokines that were measured in the intestine adjacent to the inflammatory lesion ${ }^{33,34}$. No protective effects were seen within the lesion itself ${ }^{33}$, which might reflect the powerful effect of locally applied TNBS. Furthermore, inflammatory markers of colitis (for example, histological score and colonic cytokine content) were only modestly reduced by $\mathrm{VNS}^{34}$. In a small pilot trial, seven patients with ileo-colonic Crohn's disease were implanted with a cervical vagus nerve electrode and stimulated continuously for 6 month ${ }^{65}$. The device itself was well tolerated, with only some reports of throat pain and voice alterations during $\mathrm{ON}$ phases. Two patients with a more severe form of Crohn's disease left the study owing to worsening of symptoms; however, the remaining five patients achieved clinical and endoscopic remission, including one patient who remained in remission for 42 months after surgery and no longer required azathioprine. These results, together with the mild protective effects of VNS in rats with TNBS-induced colitis ${ }^{33}$ suggest that VNS therapy is more appropriate for patients with mild or less-advanced Crohn's disease. Additional VNS clinical trials with higher patient numbers are currently being conducted ${ }^{66,67}$, but without a clear understanding of the mechanisms activated by VNS determining why some patients respond to therapy is difficult.

\section{SNS for IBD}

In a single patient with proctitis, sacral nerve stimulation (SNS) to relieve faecal incontinence improved the state of the rectal mucosa ${ }^{68}$. Endoscopic and histological scores of mucosal integrity and disease activity index improved over an 18-month period of SNS treatment and permeability (leakiness) of the mucosa, measured in mucosal biopsies, decreased. In a rat TNBS model of ulcerative colitis, SNS improved disease score, improved mucosal condition and reduced inflammatory cytokines significantly in stimulated compared with nonstimulated animals ${ }^{69}$. The mechanisms by which SNS improves mucosal integrity and reduces inflammation are not known. Similar to VNS for the treatment of IBD, determining whether an efferent effect or an effect through activation of sacral nerve afferents is involved is difficult for SNS and has been even less investigated than for VNS. SNS stimulates both motor and sensory pathways, so both hypotheses are possible.

histopathologist that quantifies

damage to the colon.

Nicotinic receptors

A class of cell surface receptors

that bind and mediate cellular

effects of acetylcholine. involve interactions between a pathological sensitization and activation of sympathetic (noradrenergic) motilityinhibiting pathways and a more slowly developing local inflammation $^{61,62,64}$. Gastrointestinal adverse effects of opioid analgesics can also contribute to the slowing of intestinal transit ${ }^{64}$. Previous therapeutic approaches to preventing and treating this condition have used drugs to target inflammatory processes (for example, nonsteroidal anti-inflammatory drugs), inhibitory neural pathways (antiadrenergics) or motility-stimulating mechanisms (for example, metoclopramide) ${ }^{70}$.

Studies in a rat model of ileus caused by manipulation of the small intestine have demonstrated that VNS might be effective in preventing and treating this dis$\operatorname{order}^{53,71}$. The efficacy of stimulating vagal pathways to treat ileus has also been confirmed in a rat model in which a pharmacological stimulation - injection of a thyrotropin-releasing hormone agonist into the CNS - was used to activate the vagus nerve ${ }^{72}$. Vagal stimulation prevented the slowing of gut transit, presumably via inhibition of inflammation, as it also inhibited infiltration of myeloperoxidase-expressing immune cells into the gut ${ }^{53,71}$. This effect seemed to be dependent on expression of both nicotinic receptors and STAT3 in gut-resident macrophages ${ }^{53,54}$, indicating a dependence on vagal cholinergic innervation of macrophages as mentioned earlier. However, another study, in which ileus was also induced in rats by intestinal handling, suggested that indirect electrical stimulation of vagal pathways could prevent ileus without inhibiting inflammation ${ }^{73}$.

Ileus is an acute condition that usually resolves within $\sim 8$ days, so the utility of an implanted stimulator is doubtful. However, given the long-lasting anti-inflammatory effects of $\mathrm{VNS}^{74}$, stimulation during abdominal surgery is potentially a useful approach to prevent postoperative ileus. Results in patients undergoing colorectal surgery are promising and show that just 2 minutes of VNS at $5 \mathrm{~Hz}$ or $20 \mathrm{~Hz}$ before and after the colorectal resection reduces systemic pro-inflammatory cytokines, IL- 6 and IL-8, compared to sham stimulation $(P<0.05)^{71}$ (TABLE 1).

\section{Neuromodulation for obesity \\ Epidemiology and current treatments}

Obesity treatment is among the most developed applications for bioelectric neuromodulation. Various devices have been trialled ${ }^{5}$ and one of these (vBloc Therapy Maestro system (EnteroMedics)) has gained FDA approval. The high prevalence and rapid increase in obesity and obesity-related illnesses worldwide, together with the current dearth in treatments that are safe and effective in the long term, is driving the intense current interest in devices for the treatment of obesity. Obesity and related conditions are a considerable public health burden in both developed and developing countries. In 2013 , up to $65 \%$ of adults in the USA qualified for weight loss intervention treatment ${ }^{75}$, and direct medical costs per patient in 2014 were estimated to be US $\$ 1,901$ per year or $\$ 149.4$ billion overall $^{76}$. A major difficulty in the treatment of obesity is that, following major weight loss, dysregulation of digestive hormones and the compensatory decrease in metabolism leads to increased sensations of hunger and food-seeking behaviour ${ }^{77-79}$. 
Excess weight loss

(EWL). A common metric for reporting loss of excess body weight, calculated as $100 \% \times$ (weight loss / excess weight at beginning of treatment); excess weight is defined as the difference between the patient's weight and the body weight if BMI were 25
These changes are contributing factors in the failure of non-invasive and lifestyle interventions for obesity, which are essentially ineffective in the long term ${ }^{79}$. Currently, bariatric surgery is regarded as the only effective treatment for inducing sustained weight loss ${ }^{80}$. The procedure reduces total body weight by $20-35 \%$ by inhibiting appetite, reducing the patient's preference for high-calorie fatty and sweet food ${ }^{81}$ and increasing energy expenditure by altering hormonal signalling from the gut $^{82}$. However, despite the long-term proven effectiveness of bariatric surgery, this approach has major drawbacks, including surgical costs, a high rate of complications ( $17 \%$ in one report, including infections, perforation and adhesions), relatively frequent (7\%) need for re-operation and even reported fatalities ${ }^{83}$, meaning that $<1 \%$ of eligible patients undergo this treatment. This low treatment rate supports the need for new treatment options for obesity, such as bioelectric neuromodulation. Neuromodulation devices for the treatment of obesity have been used to change activity in the vagus nerve directly or have been applied to the gastric surface.

\section{Influence of vagal pathways on appetite}

Vagal afferent fibres are the major conduit through which the nutrient status of the gastrointestinal tract is relayed to the brain in order to influence food intake ${ }^{3}$. Nutrients within the gut lumen (for example, carbohydrates, fats and protein fragments) activate enteroendocrine cells to release satiety hormones, the main ones being cholecystokinin, peptide YY and GLP-1 (REF. ${ }^{6}$ ). These appetite-affecting hormones act on vagal sensory nerve endings ${ }^{6,13}$. A signal for release of the appetite-stimulating hormone ghrelin is a decline in circulating glucose levels ${ }^{84}$. Vagal mechanosensitive afferent fibres also give rise to intraganglionic laminar endings and intramuscular arrays within the stomach wall that signal both tension and stretch ${ }^{11,12}$. Because these structures respond to both passive stretch and contraction of the stomach, they can signal gastric volume and contribute to sensations of fullness ${ }^{11,12}$. Vagal afferents from the liver also signal metabolic state. Thus, both satiety and hunger signals are communicated to the brain via vagal afferents.

\section{Vagal nerve blocking for obesity}

Cutting the abdominal vagus causes a decrease in food intake in individuals with healthy weight and those with obesity ${ }^{85,86}$, and in laboratory rats ${ }^{87}$. Owing to the historical use of this procedure in the treatment of gastroduodenal ulcers, its consequences in humans are well described and include diarrhoea, malabsorption and malnutrition ${ }^{85}$. In one small trial, abdominal vagotomy was used as a specific obesity treatment in 21 patients with morbid obesity ${ }^{86}$. Substantial weight loss occurred in a subset of patients $(n=14$; weight loss of $20 \pm 4 \mathrm{~kg}$ ) but was not sustained and could potentially be due to the reported large, transient placebo effect of similar procedures ${ }^{86}$. Nevertheless, these patients did report a "characteristic lack of hunger sensations", suggesting that the net effect of vagal activity is to stimulate eating ${ }^{85,86}$. These observations were the foundation for the hypothesis that blocking afferent vagus nerve signalling might promote satiety and weight loss. The vBloc Therapy Maestro system aims to recapitulate the effect of truncal vagotomy to reduce food intake by applying a blocking electrical stimulus (high-current, high-frequency stimulation for up to 12 hours per day; TABLE 1) to both the posterior and anterior vagus nerves, at the level of the gastro-oesophageal junction (FIG. 3). The device is FDA-approved for individuals with a BMI of $35-45 \mathrm{~kg} / \mathrm{m}^{2}$.

Early trials of vBloc had highly variable results ${ }^{88,89}$ and were poorly controlled, including one trial in which low levels of stimulation, which might still be above the threshold, were applied in the control group ${ }^{90}$. The ReCharge trial published in 2014 included a control group that had a non-stimulated sham device implanted ${ }^{89}$. In this trial, patients treated with vBloc experienced significantly higher excess weight loss (EWL) than the sham-device group $(26.1 \%$ versus $16.9 \% ; P=0.002)$. However, the study failed to reach its co-primary end points of $\geq 55 \%$ of patients treated with vBloc achieving a $20 \% \mathrm{EWL}$ and $\geq 45 \%$ achieving a $25 \%$ weight loss. Subsequent studies showed that patients treated with vBloc sustained EWL at 18-24 months (21-23\% EWL, equalling $8 \%$ body weight at the time of implantation), whereas the control group, which was unblinded at this stage of the study, regained weight ${ }^{91,92}$. Thus, although the effect of placebo intervention on weight loss could compromise the conclusions of these clinical trials, the data show that vBloc therapy can produce a modest, yet sustained weight loss ${ }^{91}$. Adverse events included pain at the subcutaneous site where the pulse generator was implanted, heartburn, dyspepsia, abdominal pain, swallowing defects and nausea and belching, but these were generally mild and self-resolving; severe adverse events were rare ${ }^{93}$. Furthermore, the efficacy of this regime in blocking action potentials in the human vagus has not been directly demonstrated.

\section{VNS for obesity}

Surprisingly, given the effects of vagotomy and vagal block described above, animal studies show that electrical excitation of the vagus nerve reduces food intake and limits weight gain. Bilateral VNS at the level of the gastro-oesophageal junction caused a gradual but sustained weight loss in non-obese dogs $(n=4)$ compared to pre-stimulation weight ( $27 \%$ decrease; $P=0.05)^{94}$. A number of subsequent studies show that application of VNS with low frequency $(0.1-1 \mathrm{~Hz})$ and unusually long pulse width $(10-100 \mathrm{~ms})$ results in a decrease in food intake and decrease in weight gain for normal and diet-induced obese rats ${ }^{93,95-97}$. In a study in diet-induced obese mini-pigs, VNS with increased frequency $(30 \mathrm{~Hz})$ and reduced current pulse width $(0.5-1 \mathrm{~ms})$ was also effective in reducing weight gain, food consumption and intake of sweet food (when subjected to a three-choice meal test $)^{98}$. PET scans of pig brains that received VNS at $30 \mathrm{~Hz}$ and $1 \mathrm{~ms}$ pulse width showed activation of central dopaminergic reward areas (cingulate cortex, caudate nucleus, putamen). The authors concluded that the activity of vagal efferents was unlikely to be involved in the observed effects, as no changes in gastric compliance or gastric emptying were seen ${ }^{99}$. 


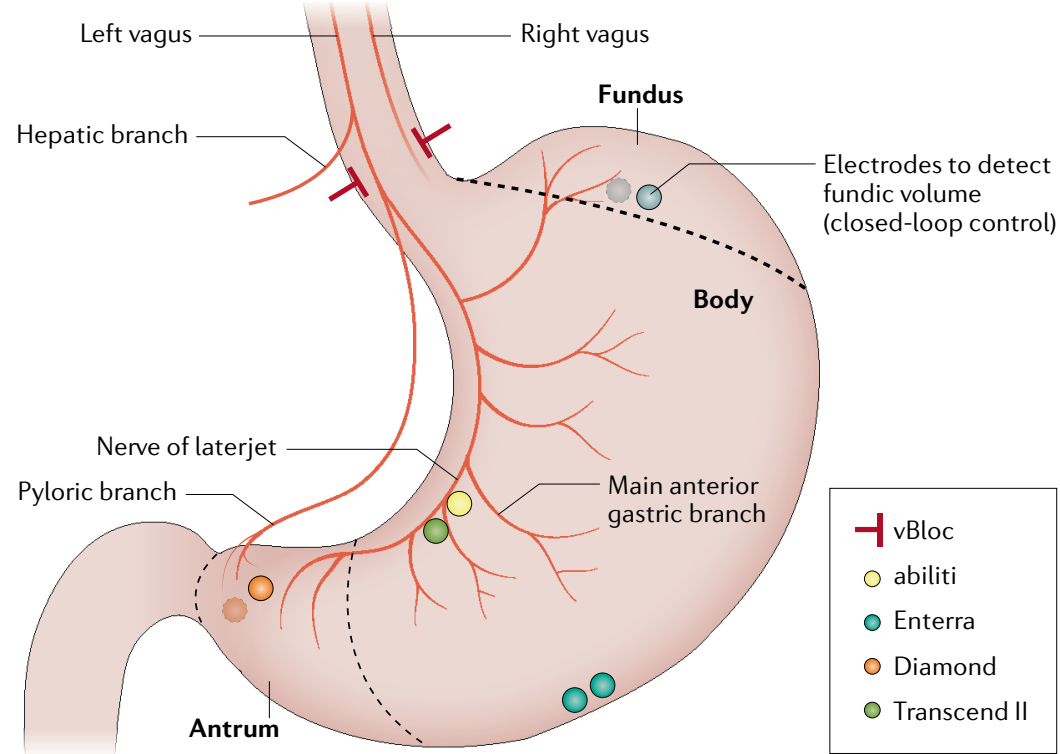

Fig. 3 | Gastric sites of bioelectric neuromodulation. Neuromodulation therapies, including gastric electrical stimulation, have been applied to various sites in the stomach and subdiaphragmatic vagus in patients. vBloc is applied bilaterally to the vagus nerves with a high-frequency current to prevent afferent signalling that induces feeding from reaching the brain. Other devices are intended to stimulate nerves or muscles and are placed on the gastric surface. The DIAMOND and Transcend II devices both use a closed-loop control. These devices and the abiliti device aim at weight reduction and the DIAMOND device is also being tested to improve glycaemic control. The Enterra device targets postprandial nausea and discomfort of gastroparesis.

In summary, vagal nerve blocking $(5000 \mathrm{~Hz})$ in patients is associated with modest weight loss and in animal models VNS is also effective. No specific clinical trial in individuals with obesity using VNS has been conducted to date. However, a retrospective assessment of patients with epilepsy and depression implanted for 2 years with a VNS device showed that stimulation therapy $(30 \mathrm{~Hz}$, up to $1.25 \mathrm{~mA}, 500 \mu$ s pulse width) resulted in $5-10 \%$ reduction in total body weight ${ }^{100,101}$ (TABLE 1).

Nausea, hedonic eating, satiety and hunger are inter-related. For example agents that increase appetite also have anti-nauseant actions, and nausea and satiety increase in paralle ${ }^{84,102}$. Vagal stimulation can activate satiety and hunger pathways and pathways that increase or decrease nausea ${ }^{12,84}$. Moreover, activity in these pathways depends on both physiological and emotional states ${ }^{13}$. Hence, varying outcomes of VNS and differing effects of vagal block in different patients are not surprising. Diet-induced changes in responses to vagal afferent activity would be expected to alter the effectiveness of VNS. In particular, a decrease in the release of the anorectic cocaine-regulated and amphetamine-regulated transcript protein (also known as CART peptide) in the nucleus tractus solitarius from vagal afferent terminals, which results in a reduction in satiety after ingestion of a calorie-rich diet, might lead to over-eating and obesity ${ }^{3}$. VNS might have an additional benefit in individuals with obesity, as obesity results in chronic low-grade inflammation that is involved in the pathogenesis of obesity-related insulin resistance and type 2 diabetes mellitus ${ }^{103}$. Thus, vagal stimulation
Eating for pleasure that is not

need for nutrient. necessarily associated with parameters could exist that reduce both obesity and inflammation-induced effects.

\section{GES for obesity}

Gastric electrical stimulation (GES) is a term used to describe electrical stimulation to the surface of the stomach or to vagal branches on the gastric surface. As explained later, the clinical effects of GES are now recognized to be due to nerve stimulation ${ }^{104}$. Devices for GES are proposed to be a safer and more reversible treatment for obesity than bariatric surgery ${ }^{105}$. Three implantable GES devices have been trialled for obesity: Transcend (Medtronic); DIAMOND (also known as Tantalus; MetaCure; now discontinued); and abiliti (IntraPace). These devices are implanted laparoscopically and in some cases have recording electrodes at the gastric fundus to detect food ingestion and control the delivery of stimulation ${ }^{105-108}$ (FIG. 3; TABLE 1). The earliest device, the Transcend system, had stimulating electrodes placed in the gastric antrum, which were claimed to act as pacemakers for gastric contractions ${ }^{5,109,110}$. Antral electrodes were shown to enhance antral contractions in rats, but the stimulus needed to be synchronized with endogenous rhythmic contractions ${ }^{111}$. However, a stimulation level sufficient to stimulate the gastric muscle will also activate nerves, which are the likely substrate for modulation of obesity ${ }^{1}$.

Studies testing the effect of GES on inducing weight loss in patients with obesity had highly variable results. Initial open-label pilot studies showed beneficial weight loss ${ }^{112,113}$, but a large-scale randomized, placebo-controlled, double-blind clinical trial of the Transcend device (the SHAPE trial) found no differences in EWL between GES-treated patients and the sham (implanted but nonstimulated) group (11.8\% versus $11.7 \%$, respectively $)^{106}$. To improve on this technology, subsequent generations of GES devices (DIAMOND and abiliti) used a closed-loop feedback system to control the delivery of GES. Electrodes are placed on both the anterior and the posterior surface of the fundus (transgastrically) to detect increased gastric volume as the stomach fills and provide feedback to adjust GES appropriately (via electrodes placed on the antrum, similar location to the Transcend device $)^{111,112}$. In clinical trials, the DIAMOND device was effective in improving glycaemic control in a blinded crossover study in patients with obesity with type 2 diabetes mellitus, and GES-treated patients experienced significant $(\geq 5 \%)$ mean total body weight loss within 12 months ${ }^{107,114,115}$. In some patients, a small decrease in weight persisted for 3 years ${ }^{115}$. However, these GES trials did not include a control group. The third-generation GES device abiliti also has closed-loop control over the delivery of GES. In addition to electrodes placed in the fundus, this device's stimulation targets the anterior vagal branches at the lesser curve of the stomach (known as the crow's foot), as well as having an inbuilt 3D accelerometer to generate telemetry data of physical activity. Data from open-label trials of the device are promising (30-60\% EWL at 12 months $)^{105,108}$, but these studies also lack control groups. GES is generally considerably less effective in inducing weight loss than bariatric surgery, 
Slow waves

Slow oscillation in the membrane potentials of muscle cells that can lead to regular contractile activity.

Chagas disease A disease caused by infection with the protist Trypanosoma cruzi that can result in degeneration of colonic enteric neurons.

Onuf's nucleus

A gathering of nerve cells in the sacral spinal cord that innervate the pelvic floor, including the external anal sphincter

Spinal cord injury Injury to the spinal cord sufficient to cause clinically recognizable deficits of sensory or motor functions. but the technology is markedly safer and associated with fewer and less-severe adverse events ${ }^{116}$. Taken together, GES is a potentially promising technology that might have beneficial effects on weight loss and is a safer option than bariatric surgery. However, owing to the lack of data from placebo-controlled clinical trials, the efficacy of GES for obesity is still uncertain.

\section{GES for the nausea of gastroparesis}

Gastroparesis is a chronic disorder of delayed gastric emptying in the absence of obstruction that is associated with postprandial distress, nausea and vomiting. Pharmaceutical treatments have proven ineffective $\mathrm{e}^{117,118}$. Gastroparesis is most commonly associated with diabetes, but is classified as idiopathic in $\sim 40 \%$ of patients ${ }^{119}$. Almost all patients with gastroparesis ( $>90 \%)$ experience nausea ${ }^{119}$. Gastric emptying is dependent on the conduction of slow waves of electrical activity that generate annular contractions of the stomach that propel contents towards the pyloric sphincter. As each contraction arrives, a small aspirate of gastric content is pushed past the sphincter into the duodenum ${ }^{120}$. The original aim of GES was to enhance these slow waves $^{1,121}$, but it is now apparent that the stimulation activates gastric afferent fibres and, therefore, conveys signals to the $\mathrm{CNS}^{104}$. In fact, the standard parameters of the system approved in the USA (Enterra system, Medtronic) are brief high-frequency trains $(14 \mathrm{~Hz}$ for $0.1 \mathrm{~s}$, repeated once per $5 \mathrm{~s}$ ) with short pulse widths $(330 \mu \mathrm{s})$ and low-energy stimulation $(5 \mathrm{~mA})^{122}$ (TABLE 1). These parameters are unlikely to stimulate muscle and do not match the slow wave frequencies of 3 waves per minute $^{120}$. In order to entrain slow waves, pulse widths of $10-600 \mathrm{~ms}$ and frequencies lower than $\sim 4$ per minute are required, but the high energy consumption necessary for such gastric pacing would make these devices impracti$\mathrm{cal}^{1}$. The stimulation is delivered by wire electrodes that are pushed into the external musculature of the anterior aspect of the stomach. The electrodes are placed $1 \mathrm{~cm}$ apart $\sim 10 \mathrm{~cm}$ from the pylorus towards the greater curvature in the gastric corpus (FIG. 3).

The mechanisms by which GES might be effective for the treatment of the nausea of gastroparesis have not been fully clarified to date. Electrical stimulation applied to the gastric corpus, using parameters that stimulate vagal afferent fibres, reduces both nausea and postprandial satiety ${ }^{121,122}$. Afferent fibres from the corpus include those that innervate the ghrelin-producing enteroendocrine cells of this region and, in both animal models and humans, ghrelin exerts its effects by activating vagal afferents $^{123,124}$. Appetite stimulation (reduction in satiety) and anti-nauseant effects are positively correlated and both are mediated by ghrelin ${ }^{84}$. The other enteroendocrine cells in the gastric corpus are 5-HT cells that promote nausea and increase satiety and somatostatin cells, which have little effect on these parameters ${ }^{16}$. Thus, we hypothesise that the symptomatic relief of gastroparesis by GES is due to the artificial activation of gastric afferents that are normally physiologically activated by ghrelin. One review and one meta-analysis of GES for the treatment of gastroparesis published in the past 2 years have identified benefit in only some trials ${ }^{104,117}$. In the meta-analysis,
16 open-label, follow-up trials showed total symptom score reductions (nausea or nausea and vomiting, postprandial discomfort), but 5 studies comparing symptoms with stimulation randomly on or off (the patients serving as their own controls) showed no difference between the on and off conditions ${ }^{117}$. An analysis of 49 studies in patients with gastroparesis showed a significant correlation $(P<0.01)$ between initial symptom severity and extent of reduction in symptoms in those treated with $\mathrm{GES}^{117}$. Data on the spontaneous resolution of symptoms for comparison was not available. The extent of postprandial satiety experienced by patients with gastroparesis was reduced by gastric electrical stimulation in 10 of 10 studies $^{117}$. In one study, satiety, evaluated after 12 months of treatment, significantly decreased $(P<0.001$; stimulus on during the final 6 months $)^{125}$. Taken together, GES shows promise as an effective treatment of gastroparesis, but requires further validation.

\section{Nerve stimulation for colorectal dysfunction Diseases affecting colorectal function}

Faecal continence and defecation are under voluntary control in humans, who can, in healthy conditions, decide when to retain or release bowel contents. However, the control is impaired in patients with disorders, including Parkinson disease, multiple sclerosis, Chagas disease, spinal trauma, meningocele, constipation of ageing, Hirschsprung disease, pelvic floor disorders, prolapse, childhood constipation and idiopathic conditions, that are associated with diarrhoea, faecal soiling or constipation. The neural circuitry includes cortical control centres, an intermediate integrative centre in the pons, the lumbosacral defecation centres and the ENS ${ }^{18}$ (FIG. 4). The final effects of these pathways have both autonomic and somatic components. For defecation, activation of autonomic pathways that synapse within the enteric nervous system within the bowel wall is required. In patients with Hirschsprung disease, in whom the ganglia of the ENS in the distal bowel have failed to develop but all other tissue components are intact and functional, severe constipation occurs and the newborn child will die if the region lacking the ENS is not removed ${ }^{126}$. Similar absence of enteric neurons in the distal bowel is also lethal in other species, including horses, rats and mice $^{127}$. Degeneration of colonic enteric neurons in Chagas disease causes failure of colorectal propulsion and development of megacolon in adults, similar to the signs associated with Hirschsprung disease in children ${ }^{128}$.

In addition to the essential role of the ENS, defecation involves the relaxation of the external anal sphincter, which is controlled by somatic pathways through Onuf's nucleus. Faecal continence depends on the control of defecation pathways and can be aided by contraction of the external anal sphincter (striated muscle). Spinal cord injury (SCI) is a condition that illustrates the roles of voluntary control of colorectal function. In patients with SCI, the defecation centres in the lumbosacral spinal cord are not subject to voluntary control; hence, these patients are unable to empty the bowel when defecation is convenient and leakage of bowel contents occurs at inappropriate times ${ }^{129-132}$. Bowel dysfunction is one of the most common secondary 
effects of SCI, and is highly inconvenient and embarrassing for many patients owing to anal leakage and difficulties with assisted bowel emptying ${ }^{133-135}$. Disturbances of bowel function occur in $>80 \%$ of individuals with $\mathrm{SCI}^{136}$. Failure of normal neural control of the bowel can have considerable adverse effects, including impaction, haemorrhoids, rectal bleeding, prolapse, formation of anal fissures and chronic constipation leading to megacolon requiring operative diversion ${ }^{130}$.

Parkinson disease is another CNS disorder associated with loss of control of colorectal function. Chronic constipation is a dominant manifestation in $80-90 \%$ of patient ${ }^{137}$, and occurs 2-4 times more frequently in patients with Parkinson disease than in age-matched and sex-matched controls ${ }^{138,139}$. In general, constipation is a considerable problem, especially in elderly individuals, as it occurs in $20-25 \%$ of those aged $>65$ years and in $\sim 50 \%$ of those in nursing homes ${ }^{140}$. Constipation is also an important concern for children, as up to $30 \%$ of school-aged children can be affected (study population comprising individuals in the UK, USA, Australia, Canada and China $)^{141}$. New drugs for treating constipation include $5-\mathrm{HT}_{4}$ receptor agonists, such as tegaserod and prucalopride, which stimulate motility, and chloride channel agonists, such as lubiprostone, which enhance fluid secretion, but they have not become preferred treatments owing to their low efficacy and adverse effects $^{142}$. The most common treatment remains osmotic laxatives, but these also have adverse effects ${ }^{143}$. To treat the severe constipation occurring in patients with SCI, the most common approaches to bowel management are manual emptying and use of osmotic laxatives ${ }^{131}$. Thus, colorectal dysfunction remains poorly treated, similar to other gastrointestinal disorders. In the search for better treatments for colorectal disorders, a range of bioelectric neuromodulation methods have been investigated, many of which are promising. Here, we summarize those that are furthest developed and discuss their mechanisms of action.

\section{SNS for faecal incontinence}

Pelvic nerve stimulation, generally referred to as sacral nerve stimulation (SNS) because the autonomic outflows (pelvic nerves) from the lower spinal cord run initially in the sacral nerves ${ }^{144}$, has been used for $>20$ years to treat faecal incontinence ${ }^{145}$. Other methods of treatment, such as lifestyle and/or behavioural interventions, laxatives, loperamide, training in toileting techniques and biofeedback, are generally ineffective ${ }^{140}$.

The pelvic nerves emerge from the most caudal lumbar nerve roots (L5 in humans, L6 in rats) and the sacral nerve roots (S1 to S4). They supply the left colon, sigmoid colon and rectum in humans (collectively called the colorectum) and the distal colon and rectum in other species. SNS works for faecal incontinence by contracting the external anal sphincter ${ }^{144}$. Numerous studies have demonstrated effectiveness of SNS in the treatment of faecal incontinence in adults ${ }^{146,147}$.

\begin{tabular}{|lc|}
\hline - Second-order sensory neuron & $\bullet$ Enteric \\
- Pathways to external & neuron \\
anal sphincter & $\rightarrow$ Afferent \\
- Autonomic pathways & neuron \\
to colorectum & ending \\
- Primary sensory neuron & A Afferent \\
- Voluntary control from cortex & neuron \\
\hline
\end{tabular}

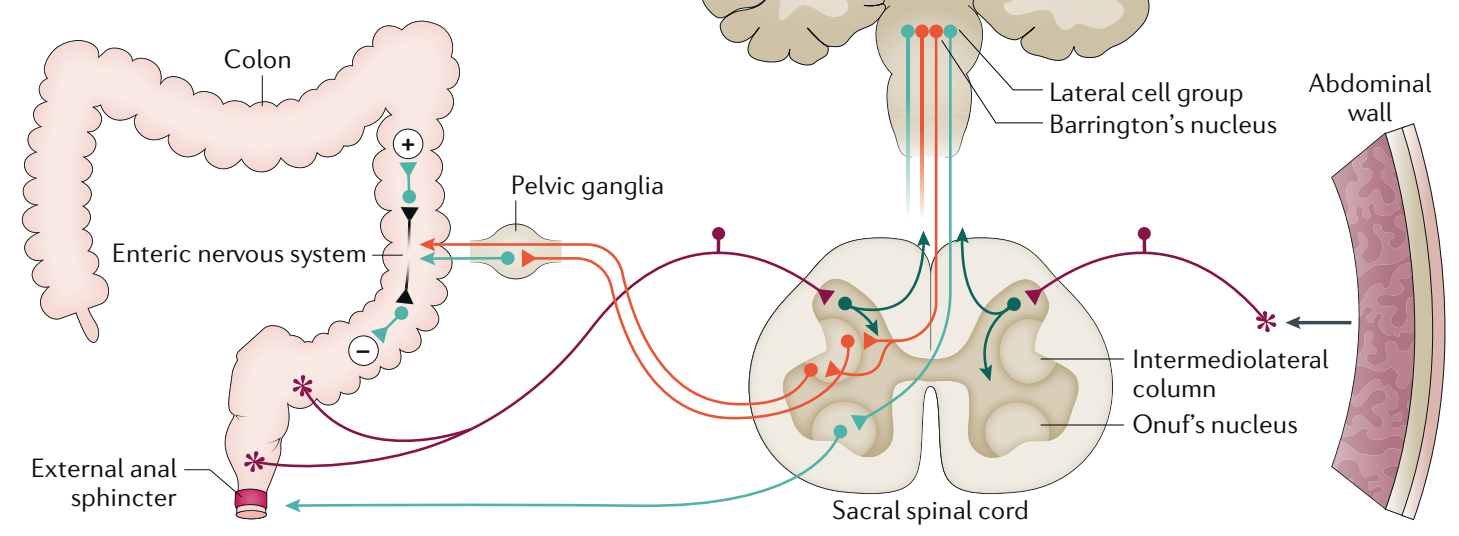

Fig. 4 | Nerve pathways for voluntary control of defecation and faecal continence. Cortical centres that regulate voluntary control provide inputs that either inhibit or enhance excitability of neurons in the brain stem via a medial nucleus (Barrington's nucleus), through which autonomic pathways to the distal colon and rectum are activated, and a lateral cell group that controls the external anal sphincter. The medial group of neurons projects to the spinal defecation centre in the intermediolateral column at the sacral S1 level. This centre in turn connects with intrinsic reflex pathways of the enteric nervous system via the pelvic ganglia. Afferent (sensory) neurons that detect pressure and mucosal irritation in the colon contribute to defecation urge, and neurons that sense pressure in the abdominal cavity enhance defecation. These sensory neurons connect to second-order afferent neurons that make local connections in the spinal cord and provide sensory information to the pons and cortex. Descending neurons from the lateral cell group synapse in Onuf's nucleus on motor neurons that supply the external sphincter. Adapted from REF. ${ }^{18}$, Springer Nature Limited. 
SNS is increasingly used to manage faecal incontinence because of the absence of other effective therapies ${ }^{140}$, electrode insertion is fairly straight-forward, complications are rare and success rates of $50-70 \%$ are obtained $^{146}$. The device consists of an electrode probe that is inserted unilaterally through the third sacral foramen and has four electrodes that are positioned close to the third sacral nerve. The electrode probe is connected by a lead to a fully implanted subcutaneous pulse generator and can be programmed and switched on and off through an external controller.

As a standard, based on parameters used in one of the original studies ${ }^{145}$, the stimulus frequency is $14 \mathrm{~Hz}$ with a pulse width of $210 \mu$ s (TABLE 1). The stimulation amplitude is set according to the patient's perception of stimulation in the perineum or anus. Few alternative parameters have been investigated ${ }^{1}$, but one study reported an improved effect with $31 \mathrm{~Hz}$ stimulation in patients with sustained loss of effectiveness using $14 \mathrm{~Hz}$ stimulation $^{148}$. In this group, incontinence episodes dropped from 11.7 to 4.8 per 3 weeks $(P<0.01)$, suggesting that $31 \mathrm{~Hz}$ stimulated the nerves more effectively. As a method for presurgical evaluation, temporary percutaneous nerve evaluation (TPNE) has been introduced. This approach involves the temporary implantation of an electrode on sacral nerves under local or general anaesthesia ${ }^{149}$. Correct positioning is evaluated by patients experiencing sensation and/or visible contraction of the pelvic floor. TPNE enables assessment of the potential effectiveness of SNS before an expensive and more invasive electrode and stimulator insertion is performed. In patients who had both positive motor and positive sensory responses to TPNE, $81-86 \%$ were successfully treated when the permanent stimulator was implanted, whereas the success rate was $68 \%$ in those who had a positive sensory response only ${ }^{150}$.

\section{SARS for constipation}

Sacral anterior root stimulation (SARS) is a modification of SNS for treating constipation. Unmodified SNS, although tested as a therapy for $>30$ years, is not reliable and has not become a treatment of choice ${ }^{151,152}$. The SARS procedure involves severing the dorsal roots and placing stimulating electrodes on the ventral roots at S2 to S4 (REF. ${ }^{153}$ ). The method was introduced in 1976 and is commonly used to treat neurogenic urinary bladder disorders, initially without the dorsal roots being severed, but it has also been used to treat bowel dysfunction in individuals with SCI ${ }^{153,154}$. In a study in $>200$ patients with constipation as a consequence of SCI (median age 49 years), SARS increased the frequency of defecation, reduced bowel emptying times and reduced the use of oral laxatives or suppositories and digital evacuation compared with patients pre-implant ${ }^{153}$.

\section{TIENS for constipation}

Transcutaneous interferential electrical nerve stimulation (TIENS) is a method in which oscillating currents, generally $1-4 \mathrm{kHz}$ sinusoidal currents, are passed between pairs of surface electrodes (FIG. 1). A common method is to use two pairs of electrodes placed approximately at right angles ${ }^{155}$.
The theory is that a heightened electrical field strength occurs where the currents from each of the electrode pairs cross, which stimulates nerves in that area ${ }^{156}$. One particular advantage of TIENS over other nerve stimulation procedures is that it is non-invasive. The method has been most commonly used to treat pain, but its effectiveness and the probability of placebo effects is being debated ${ }^{157}$. This method or variants have been used to treat slow-transit constipation and functional constipation, as well as IBS and functional dyspepsia ${ }^{155}$. The effects of TIENS on constipation in 15 studies have been reviewed in 2018 (REF. ${ }^{155}$ ). All studies showed relief of constipation with TIENS. Most of the 15 studies have been performed in children, and most of these at a single centre ${ }^{158}$. In the largest of these studies, two electrodes were placed on the anterior abdominal wall between the umbilicus and the costal margin and two electrodes were placed on the back lateral to the spine between T9 and L2 in 39 children $^{158}$ (TABLE 1).

Currents were applied through one anterior and one posterior electrode at frequencies of $4 \mathrm{kHz}$ to achieve diagonal crossing of the currents ${ }^{155}$. Increased continence and decreased soiling were observed in $73 \%$ of patients, who acted as their own controls; the effects lasted $>2$ years in $33 \%$ of patients. Manometric studies to investigate the effect and mechanism of TIENS were conducted in a group of children who had stomas at the appendix ${ }^{159}$. A perfused multiple-side-hole catheter was introduced through the stoma to record contractions of the colon. TIENS significantly increased the frequency of propagating contractions in the colon to $210 \pm 62$ per $24 \mathrm{~h}$ compared with $78 \pm 34$ per $24 \mathrm{~h}$ before application of TIENS $(P=0.008)$. In summary, findings to date indicate that TIENS has promise for the treatment of slow-transit constipation, particularly in children.

\section{Challenges for bioelectric neuromodulation}

The main challenges to successful clinical use of bioelectric neuromodulation to treat gastrointestinal tract disorders often also apply to bioelectric neuromodulation therapies in general (BOX 1).

Achieving a beneficial, clinically significant therapeutic end point is a principal problem that applies in particular to disorders that are relapsing and remitting and for which standard care cannot be withdrawn during trials. For example in Crohn's disease, withdrawing standard care in a trial of the application of VNS would put patients at risk. VNS alone might in fact result in clinically significant benefit but might not show significant additional effect when applied to patients receiving standard care. Thus, the clinical utility of a treatment such as VNS cannot always be readily tested.

Achieving disease-modifying rather than symptomatic relief is important. Generally, a therapy that treats the underlying disease is preferable to one that relieves symptoms and masks the disease. In some instances this is achievable, for example, using nerve stimulation to target the anti-inflammatory pathways to treat IBD ameliorates the disease itself. By contrast, using bioelectric neuromodulation to block the pain allows disease and tissue damage to continue to develop.

Defining an adequate nonstimulated control with which to compare the treatment group in clinical trials 
is another important obstacle. Firstly, patients who are willing to volunteer and who satisfy inclusion and exclusion criteria might not be representative of the general disease group. Secondly, whether it is ethical to surgically place an implant in a patient but not turn it on also needs to be considered. Thus, use of data from historical controls is often necessary in trial analysis, which might not have been followed and documented with the same diligence as used in a clinical trial. The lack of directly matched nonstimulated patients means that provision for placebo effects is inadequate.

Safety for devices has a dimension not shared by drug therapies. Drugs can be withdrawn, but a device that is implanted might remain as a potential source of tissue irritation or infection beyond its period of utility or the duration of a trial. Hence, ongoing patient monitoring and care are required as long as the implant remains in place, as well as a costed procedure for explant.

Optimizing stimulation parameters is one of the most vexing problems. The high number of parameters that can be changed means that thousands of variations are possible ${ }^{1,160}$. These variables include pulse shapes (for example, rectangular, biphasic or ramped), pulse durations (for example, durations from $0.1 \mathrm{~ms}$ to $300 \mathrm{~ms}$ ), train frequencies, duty cycles and the times of day or relationships to activity (for example, during sleep or after meals) at which stimuli are applied ${ }^{2}$. In the clinical setting, parameters are often 'informed guesses' based on animal studies or on extrapolation from other conditions. Adequate testing of parameters, first in animal models to refine choices and then in patients, is expensive and time consuming, but is imperative if bioelectric neuromodulation is to be optimized.

Defining and optimizing stimulation location is another obstacle to successful neuromodulation approaches. For IBD, published trials have used stimulation of the vagus in the neck, which activates fibres to the heart, lungs and larynx in addition to the intestine. The abdominal vagus supplies the intestine and its stimulation avoids fibres to the heart, lungs and larynx. In general, stimulus sites as close to the end organ as possible will optimize stimulus specificity. A further example is GES, in which the placement of electrodes on the stomach is based on false assumptions, as discussed earlier. The placement works in some patients to reduce the nausea of gastroparesis, but whether the placement is ideal is unknown.

Monitoring the effectiveness of stimulation at the stimulation site is also difficult. Therapy can fail if the stimulation does not engage the target nerve population. In this Review, we give the example of using temporary percutaneous nerve evaluation to optimize placement of electrodes that stimulate sacral nerves. Optimization might also be achieved by placing recording electrodes on the nerves, but this procedure has not been adopted in the field. Further development of methods to monitor which target nerves are actually being effectively stimulated is required.

Off-target effects of bioelectric neuromodulation need to be overcome. In the case of VNS, off-target effects include hoarseness, bradycardia and pain. Ways to avoid these effects include choice of stimulus parameters, which might need to be experimentally explored, and placement of electrodes on nerves or nerve branches at sites where off-target effects are avoided or minimized. Placement close to the end organ is predicted to reduce off-target effects, but has the disadvantage of accessing and stimulating smaller nerves that might be damaged in the process.

The maintenance of neuromodulation effectiveness over time requires further exploration. Effectiveness might reduce or fail over time owing to several factors, including tissue growth around electrodes, device failure and plasticity of nerve circuits that adapt to the stimulation. Device failure can be minimized by rigorous bench testing and testing in animal models ${ }^{161}$.

Inter-patient variability poses another challenge to general applicability of bioelectric neuromodulation strategies. Patients seek clinical help at all disease stages, with different disease and treatment histories and sometimes after other treatments have failed. When treatments, such as bioelectric neuromodulation, are new, predicting which patients will be responsive might be impossible. However, patients with moderate disease who lack other health problems might respond best, and those with refractoriness to other treatments might be least likely to be successfully treated in device trials because their disease has progressed too far to be reversible.

Achieving feed-back (closed-loop) control is an ideal approach to bioelectric neuromodulation. This engineering approach enables measuring the parameter that is being targeted and using the information gained to automatically vary the stimulation employed. Application for digestive disorders is currently out of reach owing to a lack of appropriate implantable sensors. Digestive disorders fluctuate and arise or decline over long periods, for example, constipation develops over days and intestinal inflammation resolves over weeks. Hence, feedback currently comes from the physician through clinical examination or from patient perception. Nevertheless, in the future, methods might be developed to monitor disease intensity, convert the measure to an electrical signal and change stimulus parameters in quasi real time.

\section{Conclusions}

The extensive and accessible connections between the digestive system and the CNS, the prevalence of disorders related to the digestive system and the lack of effective alternative therapies makes the innervation of the digestive system an attractive target for bioelectric neuromodulation therapy. This approach has been investigated in several gastrointestinal disorders with variable success. Sympathetic nerve stimulation for IBD, VNS for ileus, vagal block for reduction of appetite and obesity and different types of nerve stimulation for constipation have yet to be developed to the point that they might become treatments of choice, replacing drugs, behavioural therapy or surgery. The only bioelectric treatment for a gastrointestinal disorder that is currently widely used is SNS for faecal incontinence. Part of the reason is that optimal stimuli and selection of patients most likely to benefit have not been adequately explored. Exploration of optimal parameters is demanding on patients and carers, and expensive when 
incorporated into clinical trials. Patient heterogeneity is difficult to control for when the reasons underlying the heterogeneity are unknown. Development of bioelectric neuromodulation therapies for digestive system conditions requires further development in animal models and clinical trials. A particular need is the development of a more thorough understanding of the different roles of the components of mixed nerves that are being targeted, such as the vagus and sympathetic pathways to the gut.

Published online 2 November 2018
1. Chen, J. D., Yin, J. \& Wei, W. Electrical therapies for gastrointestinal motility disorders. Expert Rev. Gastroenterol. Hepatol. 11, 407-418 (2017)

2. Bonaz, B., Sinniger, V. \& Pellissier, S. Vagus nerve stimulation: a new promising therapeutic tool in inflammatory bowel disease. J. Intern. Med. 282 46-63 (2017).

3. de Lartigue, G. Role of the vagus nerve in the development and treatment of diet-induced obesity. J. Physiol. 594, 5791-5815 (2016).

4. Willemze, R. A., Luyer, M. D., Buurman, W. A $\S$ de Jonge, W. J. Neural reflex pathways in intestinal inflammation: hypotheses to viable therapy. Nat. Rev. Gastroenterol. Hepatol. 12, 353-362 (2015).

5. Lee, P. C. \& Dixon, J. Medical devices for the treatment of obesity. Nat. Rev. Gastroenterol. Hepatol. 14, 553-564 (2017)

6. Furness, J. B., Rivera, L. R., Cho, H.-J., Bravo, D. M. $\&$ Callaghan, B. The gut as a sensory organ. Nat. Rev. Gastroenterol. Hepatol. 10, 729-740 (2013).

7. Prechtl, J. C. \& Powley, T. L. The fiber composition of the abdominal vagus of the rat. Anat. Embryol. (Berl.) 181, 101-115 (1990).

8. Kawagishi, K. et al. Tyrosine hydroxylaseimmunoreactive fibers in the human vagus nerve. J. Clin. Neurosci. 15, 1023-1026 (2008).

9. Lundberg, J., Ahlman, H., Dahlstrom, A. ¿ Kewenter, J. Catecholamine-containing nerve fibres in the human abdominal vagus. Gastroenterology $70,472-474$ (1976).

10. Ahlman, B. H. et al. Evidence for innervation of the small intestine from the cervical sympathetic ganglia. J. Surg. Res. 24, 142-149 (1978).

11. Brookes, S. J. H., Spencer, N. J., Costa, M. \& Zagorodnyuk, V. P. Extrinsic primary afferent signalling in the gut. Nat. Rev. Gastroenterol. Hepatol. 10, 286-296 (2013)

12. Berthoud, H.-R. The vagus nerve, food intake and obesity. Regul. Pept. 149, 15-25 (2008).

13. Dockray, G. J. Enteroendocrine cell signalling via the vagus nerve. Curr. Opin. Pharmacol. 13, 1-5 (2013).

14. Powley, T. L. et al. Architecture of vagal motor units controlling striated muscle of esophagus: peripheral elements patterning peristalsis. Auton. Neurosci. 179, 90-98 (2013).

15. Furness, J. B., Callaghan, B., Rivera, L. R. \& Cho, H. J. The enteric nervous system and gastrointestinal innervation: integrated local and central control. Adv. Exp. Med. Biol. 817, 39-71 (2014).

16. Furness, J. B. Integrated neural and endocrine control of gastrointestinal function. Adv. Exp. Med. Biol. 891 159-173 (2016)

17. Ness, T. J. \& Gebhart, G. F. Visceral pain: a review of experimental studies. Pain 41, 167-234 (1990).

18. Callaghan, B., Furness, J. B. \& Pustovit, R. V. Neural pathways for colorectal control, relevance to spinal cord injury and treatment: a narrative review. Spinal Cord 56, 199-205 (2018)

19. Ananthakrishnan, A. N. Epidemiology and risk factors for IBD. Nat. Rev. Gastroenterol. Hepatol. 12 , 205-217 (2015)

20. Thia, K. T., Loftus, E. V. Jr., Sandborn, W. J. $\&$ Yang, S. K. An update on the epidemiology of inflammatory bowel disease in Asia. Am. J. Gastroenterol. 103, 3167-3182 (2008).

21. Kaplan, G. G. The global burden of IBD: from 2015 to 2025. Nat. Rev. Gastroenterol. Hepatol. 12, 720-727 (2015)

22. Loftus, E. V. Jr. Clinical epidemiology of inflammatory bowel disease: Incidence, prevalence, and environmental influences. Gastroenterology 126 1504-1517 (2004).

23. Cosnes, J., Gower-Rousseau, C., Seksik, P. \& Cortot, A Epidemiology and natural history of inflammatory bowel diseases. Gastroenterology 140, 1785-1794 (2011).

24. Tsianos, E. V. \& Katsanos, K. Do we really understand what the immunological disturbances in inflammatory bowel disease mean? World J. Gastroenterol. 15 . 521-525 (2009).
25. Mow, W. S. et al. Association of antibody responses to microbial antigens and complications of small bowel Crohn's disease. Gastroenterology 126, 414-424 (2004).

26. Neurath, M. F. Cytokines in inflammatory bowel disease. Nat. Rev. Immunol. 14, 329-342 (2014).

27. Peyrin-Biroulet, L., Loftus, E. V. Jr, Colombel, J. F. \& Sandborn, W. J. Long-term complications, extraintestinal manifestations, and mortality in adult Crohn's disease in population-based cohorts. Inflamm. Bowel Dis. 17, 471-478 (2011).

28. De Cruz, P. et al. Postoperative recurrence of Crohn's disease: impact of endoscopic monitoring and treatment step-up. Colorectal Dis. 15, 187-197 (2013).

29. Gisbert, J. P., Marin, A. C., McNicholl, A. C $\S$ Chaparro, M. Systematic review with meta-analysis: the efficacy of a second anti-TNF in patients with inflammatory bowel disease whose previous antiTNF treatment has failed. Aliment. Pharmacol. Ther. 41, 613-623 (2015)

30. Ford, A. C. et al. Efficacy of biological therapies in inflammatory bowel disease: systematic review and meta-analysis. Am. J. Gastroenterol. 106, 644-659 (2011).

31. Chaparro, M. et al. Safety of thiopurine therapy in inflammatory bowel disease: long-term follow-up study of 3931 patients. Inflamm. Bowel Dis. 19, 1404-1410 (2013).

32. De Cruz, P. et al. Crohn's disease management after intestinal resection: a randomised trial. Lancet 385 1406-1417 (2015).

33. Meregnani, J. et al. Anti-inflammatory effect of vagus nerve stimulation in a rat model of inflammatory bowel disease. Auton. Neurosci. 160, 82-89 (2011).

34. Sun, P. et al. Involvement of MAPK/NF-kappaB signaling in the activation of the cholinergic anti-inflammatory pathway in experimental colitis by chronic vagus nerve stimulation. PLOS ONE 8 , e69424 (2013).

35. Ghia, J. E., Blennerhassett, P., Kumar-Ondiveeran, H., Verdu, E. F. \& Collins, S. M. The vagus nerve: a tonic inhibitory influence associated with inflammatory bowel disease in a murine model. Gastroenterology 131, 1122-1130 (2006)

36. Ji, H. et al. Central cholinergic activation of a vagus nerve-to-spleen circuit alleviates experimental colitis. Mucosal Immunol. 7, 335-347 (2014).

37. Willemze, R. A. et al. Neuronal control of experimenta colitis occurs via sympathetic intestinal innervation. Neurogastroenterol. Motil. 30, e13163-e13177 (2017).

38. Pavlov, V. A. \& Tracey, K. J. Neural regulation of immunity: molecular mechanisms and clinical translation. Nat. Neurosci. 20, 156-166 (2017).

39. Talbot, S., Foster, S. L. \& Woolf, C. J. Neuroimmunity: physiology and pathology. Annu. Rev. Immunol. 34, 421-447 (2016)

40. Martelli, D., McKinley, M. J. \& McAllen, R. M. The cholinergic anti-inflammatory pathway: a critical review. Auton. Neurosci. 182, 65-69 (2014).

41. Martelli, D., Yao, S. T., McKinley, M. J. \& McAllen, R. M. Reflex control of inflammation by sympathetic nerves, not the vagus. J. Physiol. 592, 1677-1686 (2014).

42. Holzer, P. Local effector functions of capsaicinsensitive sensory nerve endings: involvement of tachykinins, calcitonin gene-related peptide and other neuropeptides. Neuroscience 24, 739-768 (1988).

43. Holzer, P. \& Lippe, I. T. Stimulation of afferent nerve endings by intragastric capsaicin protects against ethanol-induced damage of gastric mucosa. Neuroscience 27, 981-987 (1988).

44. Holzer, P. \& Lippe, I. T. Role of calcitonin gene-related peptide in gastrointestinal blood flow. Ann. NY Acad. Sci. 657, 228-239 (1992).

45. Jacobson, E. D. Vascular mediation of gastric mucosal damage and cytoprotection. Indian J. Physiol. Pharmacol. 34, 223-234 (1990).
46. Lambrecht, N., Burchert, M., Respondek, M., Muller, K. M. \& Peskar, B. M. Role of calcitonin gene-related peptide and nitric oxide in the gastroprotective effect of capsaicin in the rat. Gastroenterology 104, 1371-1380 (1993).

47. Eysselein, V. E. et al. Calcitonin gene-related peptide in inflammatory bowel disease and experimentally induced colitis. Ann. NY Acad. Sci. 657, 319-327 (1992).

48. Mazelin, L., Theodorou, V., More, J., Fioramonti, J. \& Bueno, L. Protective role of vagal afferents in experimentally-induced colitis in rats. J. Auton. Nerv. Syst. 73, 38-45 (1998).

49. Reinshagen, $M$. et al. Protective function of extrinsic sensory neurons in acute rabbit experimental colitis. Gastroenterology 106, 1208-1214 (1994).

50. Reinshagen, M. et al. Calcitonin gene-related peptide mediates the protective effect of sensory nerves in a model of colonic injury. J. Pharmacol. Exp. Ther. 286 657-661 (1998).

51. Holzer, P. Role of visceral afferent neurons in mucosal inflammation and defense. Curr. Opin. Pharmacol. 7 , 563-569 (2007).

52. Ritter, R. C. \& Ladenheim, E. E. Capsaicin pretreatment attenuates suppression of food intake by cholecystokinin. Am. J. Physiol. 248, R501-R504 (1985).

53. de Jonge, W. J. et al. Stimulation of the vagus nerve attenuates macrophage activation by activating the Jak2-STAT3 signaling pathway. Nat. Immunol. $\mathbf{6}$, 844-851 (2005).

54. Matteoli, G. et al. A distinct vagal anti-inflammatory pathway modulates intestinal muscularis resident macrophages independent of the spleen. Gut 63 938-948 (2014).

55. De Cruz, P., Kamm, M. A., Prideaux, L., Allen, P. B. ¿ Desmond, P. V. Postoperative recurrent luminal Crohn's disease: a systematic review. Inflamm. Bowel Dis. 18, 758-777 (2012)

56. Cervi, A. L., Lukewich, M. K. \& Lomax, A. E. Neural regulation of gastrointestinal inflammation: role of the sympathetic nervous system. Auton. Neurosci. 182, 83-88 (2014).

57. Hirst, G. D. \& McKirdy, H. C. Presynaptic inhibition at mammalian peripheral synapse? Nature 250, 430-431 (1974).

58. Stebbing, M., Johnson, P., Vremec, M. \& Bornstein, J. Role of $\alpha_{2}$-adrenoceptors in the sympathetic inhibition of motility reflexes of guinea-pig ileum. J. Physiol. 534, 465-478 (2001).

59. Gabanyi, I. et al. Neuro-immune interactions drive tissue programming in intestinal macrophages. Cell 164, 378-391 (2016).

60. Vasina, V. et al. The $\beta_{3}$-adrenoceptor agonist SR58611 A ameliorates experimental colitis in rats. Neurogastroenterol. Motil. 20, 1030-1041 (2008).

61. De Winter, B. Y. et al. Effect of adrenergic and nitrergic blockade on experimental ileus in rats Br. J. Pharmacol. 120, 464-468 (1997).

62. Fukuda, H. et al. Inhibition of sympathetic pathways restores postoperative ileus in the upper and lower gastrointestinal tract. J. Gastroenterol. Hepatol. 22, 1293-1299 (2007).

63. Willemze, R. A., Bakker, T., Pippias, M., Ponsioen, C. Y $\&$ de Jonge, W. J. $\beta$-Blocker use is associated with a higher relapse risk of inflammatory bowel disease: a Dutch retrospective case-control study. Eur. J. Gastroenterol. Hepatol. 30, 161-166 (2018).

64. Bauer, A. J. Mentation on the immunological modulation of gastrointestinal motility. Neurogastroenterol. Motil. 20, 81-90 (2008).

65. Bonaz, B. et al. Chronic vagus nerve stimulation in Crohn's disease: a 6-month follow-up pilot study. Neurogastroenterol. Motil. 28, 948-953 (2016).

66. US National Library of Medicine. ClinicalTrials.gov https://clinicaltrials.gov/ct2/show/NCT02311660 (2017).

67. US National Library of Medicine. ClinicalTrials.gov https://clinicaltrials.gov/ct2/show/NCT02951650 (2017). 
68. Bregeon, J. et al. Improvement of refractory ulcerative proctitis with sacral nerve stimulation. $J$. Clin. Gastroenterol. 49, 853-857 (2015).

69. Guo, J. et al. Anti-inflammatory effects and mechanisms of sacral nerve stimulation on TNBS-induced ulcerative colitis in rats. Gastroenterology 150, S98-S99 (2016)

70. Luckey, A., Livingston, E. \& Tache, Y. Mechanisms and treatment of postoperative ileus. Arch. Surg. 138, 206-214 (2003)

71. Stakenborg, N. et al. Abdominal vagus nerve stimulation as a new therapeutic approach to prevent postoperative ileus. Neurogastroenterol. Motil. 29. e13075-e13086 (2017).

72. Yuan, P. Q. \& Tache, Y. Abdominal surgery induced gastric ileus and activation of M1-like macrophages in the gastric myenteric plexus: prevention by central vagal activation in rats. Am. J. Physiol. Gastrointest. Liver Physiol. 313, G320-G329 (2017).

73. Fang, J. F. et al. Electroacupuncture treatment partly promotes the recovery time of postoperative ileus by activating the vagus nerve but not regulating local inflammation. Scientif. Rep. 7, 39801-39815 (2017).

74. Inoue, $\mathrm{T}$. et al. Vagus nerve stimulation mediates protection from kidney ischemia-reperfusion injury through a 7nAChR+ splenocytes. J. Clin. Invest. 126, 1939-1952 (2016).

75. Stevens, J., Oakkar, E. E., Cui, Z., Cai, J \& Truesdale, K. P. US adults recommended for weight reduction by 1998 and 2013 obesity guidelines, NHANES 2007-2012. Obes. (Silver Spring) 23, 527-531 (2015).

76. Kim, D. D. \& Basu, A. Estimating the medical care costs of obesity in the United States: systematic review, meta-analysis, and empirical enalysis. Value Health 19, 602-613 (2016).

77. Leibel, R. L., Rosenbaum, M. \& Hirsch, J. Changes in energy expenditure resulting from altered body weight. N. Engl. J. Med. 332, 621-628 (1995)

78. Sumithran, P. et al. Long-term persistence of hormona adaptations to weight loss. N. Engl. J. Med. 365, 1597-1604 (2011)

79. Carvajal, R., Wadden, T. A., Tsai, A. G., Peck, K \& Moran, C. H. Managing obesity in primary care practice: a narrative review. Ann. NY Acad. Sci. 1281, 191-206 (2013)

80. Driscoll, S., Gregory, D. M., Fardy, J. M. \& Twells, L. K. Long-term health-related quality of life in bariatric surgery patients: a systematic review and metaanalysis. Obes. (Silver Spring) 24, 60-70 (2016).

81. Hofmann, W., van Koningsbruggen, G. M., Stroebe, W. Ramanathan, S. \& Aarts, H. As pleasure unfolds. Hedonic responses to tempting food. Psychol. Sci. 21. 1863-1870 (2010).

82. Miras, A. D. \& le Roux, C. W. Mechanisms underlying weight loss after bariatric surgery. Nat. Rev. Gastroenterol. Hepatol. 10, 575-584 (2013).

83. Chang, S. H. et al. The effectiveness and risks of bariatric surgery: an updated systematic review and meta-analysis, 2003-2012. JAMA Surg. 149, 275-287 (2014)

84. Sanger, G. J., Broad, J., Callaghan, B. \& Furness, J. B. Ghrelin and motilin control systems in $\mathrm{Gl}$ physiology and therapeutics. Handb Exp. Pharmacol. 239. 379-416 (2017)

85. Meyer, J. H. in Gastrointestinal Disease Pathophysiology, Diagnosis, Management (eds Sleisenger, M. H. \& Fordtran, J. S.) 757-779 (W. B. Saunders Co., 1983)

86. Kral, J. G. \& Gortz, L. Truncal vagotomy in morbid obesity. Int. J. Obes. 5, 431-435 (1981)

87. Furness, J. B. et al. Effects of vagal and splanchnic section on food intake, weight, serum leptin and hypothalamic neuropeptide $\mathrm{Y}$ in rat. Auton. Neurosci. 92, 28-36 (2001).

88. Camilleri, M. et al. Intra-abdominal vagal blocking (vBloc therapy): clinical results with a new implantable medical device. Surgery 143, 723-731 (2008).

89. Ikramuddin, S. et al. Effect of reversible intermittent intra-abdominal vagal nerve blockade on morbid obesity: the ReCharge randomized clinical trial. JAMA 312, 915-922 (2014)

90. Sarr, M. G. et al. The EMPOWER study: randomized, prospective double-blind, multicenter trial of vagal blockade to induce weight loss in morbid obesity. Obes. Surg. 22, 1771-1782 (2012)

91. Shikora, S. A. et al. Sustained weight loss with vagal nerve blockade but not with sham: 18-month results of the ReCharge trial. J. Obes. 2015 365604-365612 (2015)

92. Apovian, C. M. et al. Two-year outcomes of vagal nerve blocking (vBloc) for the treatment of obesity in the ReCharge trial. Obes. Surg. 27, 169-176 (2017).
93. Laskiewicz, J. et al. Effects of vagal neuromodulation and vagotomy on control of food intake and body weight in rats. J. Physiol. Pharmacol. 54, 603-610 (2003).

94. Roslin, M. \& M, K. The use of electrical stimulation of the vagus nerve to treat morbid obesity. Epilepsy Behav. 2, 11-16 (2001)

95. Bugajski, A J et al. Effect of long-term vagal stimulation on food intake and body weight during diet induced obesity in rats. J. Physiol. Pharmacol. 58, 5-12 (2007).

96. Krolczyk, G. et al. The effects of baclofen on the feeding behaviour and body weight of vagally stimulated rats. J. Physiol. Pharmacol. 56, 121-131 (2005).

97. Laskiewicz, J., Krolczyk, G., Zurowski, D., Enck, P. $\&$ Thor, P. J. Capasaicin induced deafferentation enhances the effect of electrical vagal nerve stimulation on food intake and body mass. J. Physiol. Pharmacol. 55, 155-163 (2004).

98. Val-Laillet, D., Biraben, A., Randuineau, G. \& Malbert, C. H. Chronic vagus nerve stimulation decreased weight gain, food consumption and sweet craving in adult obese minipigs. Appetite $\mathbf{5 5}$ 245-252 (2010)

99. Biraben, P. A., Guerin, S., Bobillier, É., Val-Laillet, D. $\&$ Malbert, C. H. Central activation after chronic vagus nerve stimualtion in pigs: contribution of functional imaging. Bull. l'Académie Vétérinaire France 161, 441-448 (2008)

100. Burneo, J. G., Faught, E., Knowlton, R., Morawetz, R. \& Kuzniecky, R. Weight loss associated with vagus nerve stimulation. Neurology 59, 463-464 (2002).

101. Pardo, J. V. et al. Weight loss during chronic, cervical vagus nerve stimulation in depressed patients with obesity: an observation. Int. J. Obes. (Lond.) 31, 1756-1759 (2007).

102. Chuang, J. C. et al. Ghrelin mediates stress-induced food-reward behavior in mice. J. Clin. Invest. 121 2684-2692 (2011)

103. Esser, N., Legrand-Poels, S., Piette, J., Scheen, A. J. $\&$ Paquot, N. Inflammation as a link between obesity, metabolic syndrome and type 2 diabetes. Diabetes Res. Clin. Pract. 105, 141-150 (2014).

104. Wo, J. M., Nowak, T. V., Waseem, S. \& Ward, M. P. Gastric electrical stimulation for gastroparesis and chronic unexplained nausea and vomiting. Curr. Treat. Opt. Gastroenterol. 14, 386-400 (2016).

105. Miras, M., Serrano, M., Duran, C., Valino, C. $\&$ Canton, S. Early experience with customized meal-triggered gastric electrical stimulation in obese patients. Obes. Surg. 25, 174-179 (2015).

106. Shikora, S. A. et al. Implantable gastric stimulation for the treatment of clinically severe obesity: results of the SHAPE trial. Surg. Obes. Relat. Dis. 5, 31-37 (2009).

107. Lebovitz, H. E. et al. Fasting plasma triglycerides predict the glycaemic response to treatment of type 2 diabetes by gastric electrical stimulation. A novel lipotoxicity paradigm. Diabet Med. 30, 687-693 (2013).

108. Horbach, T. et al. abiliti ${ }^{\circledR}$ closed-loop gastric electrical stimulation system for treatment of obesity: clinical results with a 27-month follow-up. Obes. Surg. 25, 1779-1787 (2015).

109. Lebovitz, H. E. Interventional treatment of obesity and diabetes: an interim report on gastric electrica stimulation. Rev. Endocr. Metab. Disord. 17, 73-80 (2016).

110. Mizrahi, M., Ben Ya'acov, A. \& Ilan, Y. Gastric stimulation for weight loss. World J. Gastroenterol. 18, 2309-2319 (2012)

111. Peles, S. et al. Enhancement of antral contractions and vagal afferent signaling with synchronized electrical stimulation. Am. J. Physiol. Gastrointest. Liver Physiol. 285, G577-G585 (2003).

112. D'Argent, J. Gastric electrical stimulation as therapy of morbid obesity: preliminary results from the French study. Obes. Surg. 12, S21-S25 (2002).

113. Cigaina, V. Gastric pacing as therapy for morbid obesity: preliminary results. Obes. Surg. 12, S12-S16 (2002).

114. Lebovitz, H. E. et al. Gastric electrical stimulation treatment of type 2diabetes: effects of implantation versus meal-mediated stimulation. A randomized blinded cross-over trial. Physiol. Rep. 3 e12456-e12464 (2015)

115. Lebovitz, H. E. et al. Treatment of patients with obese type 2 diabetes with Tantalus-DIAMOND ${ }^{(\mathbb{R})}$ gastric electrical stimulation: normal triglycerides predict durable effects for at least 3 years. Horm. Metab. Res. 47, 456-462 (2015)

116. Horbach, T. et al. Closed-loop gastric electrical stimulation versus laparoscopic adjustable gastric band for the treatment of obesity: a randomized 2-month multicenter study. Int. J. Obes. (Lond.) 40 1891-1898 (2016).

117. Levinthal, D. J. \& Bielefeldt, K. Systematic review and meta-analysis: gastric electrical stimulation for gastroparesis. Auton. Neurosci. 202, 45-55 (2017).

118. Pasricha, P. J., Camilleri, M., Hasler, W. L. \& Parkman, H. P. White Paper AGA: gastroparesis: clinical and regulatory insights for clinical trials. Clin. Gastroenterol. Hepatol. 15, 1184-1190 (2017).

119. Parkman, H. P. et al. Nausea and vomiting in gastroparesis: similarities and differences in idiopathic and diabetic gastroparesis. Neurogastroenterol. Motil. 28, 1902-1914 (2016).

120. Kelly, K. A. Gastric emptying of liquids and solids: roles of proximal and distal stomach. Am. J. Physiol. 239, G71-G76 (1980).

121. McCallum, R. W. et al. Gastric pacing improves emptying and symptoms in patients with gastroparesis. Gastroenterology 114, 456-461 (1998).

122. McCallum, R. W. et al. Gastric electrical stimulation with Enterra therapy improves symptoms from diabetic gastroparesis in a prospective study. Clin. Gastroenterol. Hepatol. 8, 947-954 (2010).

123. Asakawa, A. et al. Ghrelin is an appetite-stimulatory signal from stomach with structural resemblance to motilin. Gastroenterology 120, 337-345 (2001).

124. le Roux, C. W. et al. Ghrelin does not stimulate food intake in patients with surgical procedures involving vagotomy. J. Clin. Endocr. Metab. 90, 4521-4524 (2005).

125. McCallum, R. W. et al. Gastric electrical stimulation with Enterra therapy improves symptoms of idiopathic gastroparesis. Neurogastroenterol. Motil. 25, 815-824 (2013).

126. Swenson, O. Hirschsprung's disease: a review. Pediatrics 109, 914-918 (2002).

127. Furness, J. B. \& Poole, D. P. Involvement of gut neural and endocrine systems in pathological disorders of the digestive tract. J. Anim. Sci. 90 , 1203-1212 (2012).

128. Matsuda, N. M., Miller, S. M. \& Evora, P. R. B. The chronic gastrointestinal manifestations of Chagas disease. Clinics 64, 1219-1224 (2009).

129. Glickman, S. \& Kamm, M. A. Bowel dysfunction in spinal-cord-injury patients. Lancet 347, 1651-1653 (1996).

130. Lynch, A. C., Antony, A., Dobbs, B. R. \& Frizelle, F. A. Bowel dysfunction following spinal cord injury. Spinal Cord 39, 193-203 (2001).

131. Lynch, A. C. \& Frizelle, F. A. Colorectal motility and defecation after spinal cord injury in humans. Prog. Brain Res. 152, 335-343 (2006)

132. Ng, C. et al. Gastrointestinal symptoms in spinal cord inury: relationships with level of injury and psychologic factors. Dis. Colon Rectum 48, 1562-1568 (2005).

133. Snoek, G. J., Ijzerman, M. J., Hermens, H. J. Maxwell, D. \& Biering-Sorensen, F. Survey of the needs of patients with spinal cord injury: impact and priority for improvement in hand function in tetraplegics. Spinal Cord 42, 526-532 (2004).

134. Krogh, K., Perkash, I., Stiens, S. A. \& BieringSørensen, F. International bowel function extended spinal cord injury data set. Spinal Cord 47, 235-241 (2009).

135. Liu, C.-W. et al. Prediction of severe neurogenic bowel dysfunction in persons with spinal cord injury. Spinal Cord 48, 554-559 (2010).

136. Widerström-Noga, E. G., Felipe-Cuervo, E., Broton, J. G. Duncan, R. C. \& Yezierski, R. P. Perceived difficulty in dealing with consequences of spinal cord injury. Arch. Phys. Med. Rehabil. 80, 580-586 (1999).

137. Fasano, A., Visanji, N. P., Liu, L. W. C., Lang, A. E. $\&$ Pfeiffer, R. F. Gastrointestinal dysfunction in Parkinson's disease. Lancet Neurol. 14, 625-639 (2015).

138. Jost, W. H. Gastrointestinal dysfunction in Parkinson's disease. J. Neurol. Sci. 289, 69-73 (2010).

139. Lin, C.-H., Lin, J.-W., Liu, Y.-C., Chang, C.-H. \& $\mathrm{Wu}, \mathrm{R} . \mathrm{-M}$. Risk of Parkinson's disease following severe constipation: a nationwide population-based cohort study Parkinsonism Relat. Disord. 20, 1371-1375 (2014).

140. Tariq, S. H. Constipation in long-term care. J. Am. Med. Dir. Assoc. 8, 209-218 (2007).

141. Auth, M. K. H., Vora, R., Farrelly, P. \& Baillie, C. Childhood constipation. BMJ 345, e7390 (2012)

142. Shin, A. et al. Systematic review with meta-analysis: highly selective 5-HT4 agonists (prucalopride, velusetrag or naronapride) in chronic constipation. Aliment. Pharmacol. Ther. 39, 239-253 (2014). 
143. Ford, A. C. \& Talley, N. J. Laxatives for chronic constipation in adults. BMJ 345, 44-49 (2012).

144. Carrington, E. V. et al. A systematic review of sacral nerve stimulation mechanisms in the treatment of fecal incontinence and constipation. Neurogastroenterol. Motil. 26, 1222-1237 (2014).

145. Matzel, K. E., Stadelmaier, U., Hohenfellner, M. \& Gall, F. P. Electrical stimulation of sacral spinal nerves for treatment of faecal incontinence. Lancet 346 , 1124-1127 (1995).

146. Thin, N. N. et al. Systematic review of the clinical effectiveness of neuromodulation in the treatment of faecal incontinence. Br. J. Surg. 100, 1430-1447 (2013).

147. Tjandra, J. J., Chan, M. K., Yeh, C. H. \& Murray-Green, C. Sacral nerve stimulation is more effective than optimal medical therapy for severe fecal incontinence: a randomized, controlled study. Dis. Colon Rectum 51, 494-502 (2008).

148. Duelund-Jakobsen, J. et al. Randomized double-blind crossover study of alternative stimulator settings in sacral nerve stimulation for faecal incontinence. Br. J. Surg. 99, 1445-1452 (2012).

149. Mitchell, P. J., Cattle, K., Saravanathan, S., Telford, K. J. $\&$ Kiff, E. S. Insertion under local anaesthetic of temporary electrodes for sacral nerve stimulation testing is reliable and cost effective. Colorectal Dis. 13, 445-448 (2011).

150. Govaert, B., Melenhorst, J., van Gemert, W. G. \& Baeten, C. G. Can sensory and/or motor reactions during percutaneous nerve evaluation predict outcome of sacral nerve modulation? Dis. Colon Rectum $\mathbf{5 2}$, 1423-1426 (2009).

151. Varma, J. S., Binnie, N., Smith, A. N., Creasey, G. H. \& Edmond, P. Differential effects of sacral anterior root stimulation on anal sphincter and colorectal motility in spinally injured man. Br. J. Surg. 73 478-482 (1986).
152. Malouf, A. J., Wiesel, P. H., Nicholls, T., Nicholls, R. J. ¿ Kamm, M. A. Short-term effects of sacral nerve stimulation for idiopathic slow transit constipation. World J. Surg. 26, 166-170 (2002).

153. Rasmussen, M. M. et al. Sacral anterior root stimulation improves bowel function in subjects with spinal cord injury. Spinal Cord 53, 297-301 (2015).

154. Zaer, H. et al. Effect of spinal anterior root stimulation and sacral deafferentation on bladder and sexual dysfunction in spinal cord injury. Acta Neurochir. (Wien) 160, 1377-1384 (2018)

155. Moore, J. S., Gibson, P. R. \& Burgell, R. E. Neuromodulation via interferential electrical stimulation as a novel therapy in gastrointestinal motility disorders. J. Neurogastroenterol. Motil. 24 19-29 (2018).

156. Palmer, S. T., Martin, D. J., Steedman, W. M $\&$ Ravey, J. Alteration of interferential current and transcutaneous electrical nerve stimulation frequency: effects on nerve excitation. Arch. Phys. Med. Rehabil. 80, 1065-1071 (1999).

157. Johnson, M. I. Transcutaneous electrical nerve stimulation (TENS) as an adjunct for pain management in perioperative settings: a critical review. Expert Rev. Neurother. 17, 1013-1027 (2017).

158. Leong, L. C. et al. Long-term effects of transabdominal electrical stimulation in treating children with slow-transit constipation. J. Pediatr. Surg. 46 2309-2312 (2011)

159. Clarke, M. C. et al. Transabdominal electrical stimulation increases colonic propagating pressure waves in paediatric slow transit constipation. J. Pediatr. Surg. 47, 2279-2284 (2012).

160. Loerwald, K. W. et al. Varying stimulation parameters to improve cortical plasticity generated by VNS-tone pairing. Neuroscience 388, 239-247 (2018).

161. Shepherd, R. K., Villalobos, J., Burns, O. \& Nayagam, D. A. X. The development of neural stimulators: a review of preclinical safety and efficacy studies. J. Neural Eng. 15, 041004 (2018).

162. Abell, T. L. et al. A double-masked, randomized placebo-controlled trial of temporary endoscopic mucosal gastric electrical stimulation for gastroparesis. Gastrointest. Endosc. 74, 496-503 (2011).

\section{Acknowledgements}

Work on bioelectric modulation for inflammatory bowel disease is supported by the Defense Advanced Research Projects Agency (DARPA) BTO through the Space and Naval Warfare Systems Center Contract No. N66001-15-2-4060 to J.B.F., and work on gastric disorders by NIH (SPARC) grant ID\# OT2OD023847 to J.B.F., the National Institutes of Health (NIH) and the National Health and Medical Research Council of Australia. Billie Hunne is thanked for assistance in the preparation of illustrations.

\section{Author contributions}

All authors researched data for the article, made substantial contributions to discussion of the article content, wrote and reviewed/edited the manuscript before submission.

\section{Competing interests}

The authors declare no competing interests.

\section{Publisher's note}

Springer Nature remains neutral with regard to jurisdictional claims in published maps and institutional affiliations.

\section{Review criteria}

Literature searches included publications in the past 10 years. Earlier publications have been sought if they were referenced in contemporary papers. Combinations of the following search terms were used: "vagus nerve", "vagus nerve stimulation", "gastric electrical stimulation", "inflammatory bowel disease", "sacral nerve stimulation", "ileus", "gastroparesis", "faecal incontinence", "constipation", "appetite", "satiety", "nausea". Searches were conducted in Google Scholar and PubMed. 